\title{
Polyethylene as a Radiation Shielding Standard in Simulated Cosmic-Ray Environments
}

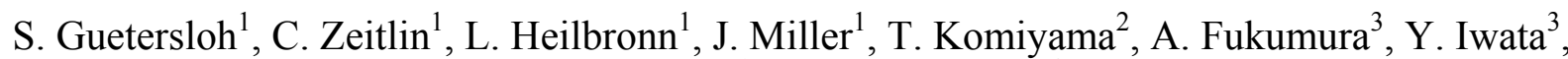 \\ T. Murakami ${ }^{3}$, M. Bhattacharya ${ }^{4}$ \\ 1. Life Sciences Division, Lawrence Berkeley National Laboratory, Berkeley, California 94720 \\ 2. Japanese Aerospace Exploration Agency, 2-1-1 Tskuba-shi, Ibaraki, Japan 305-8505 \\ 3. National Institute of Radiological Science, 4-9-1 Anagawa, Chiba, Japan \\ 4. National Space Science and Technology Center, 320 Sparkman Drive, Huntsville, Alabama, 35805
}

\begin{abstract}
Radiation risk management for human space missions depends on accurate modeling of highenergy heavy ion transport in matter. The process of nuclear fragmentation can play a key role in reducing both the physical dose and the biological effectiveness of the radiation encountered in deep space. Hydrogenous materials and light elements are expected to be more effective shields against the deleterious effects of Galactic Cosmic Rays (GCR) than aluminum, which is used in current spacecraft hulls. NASA has chosen polyethylene, $\mathrm{CH}_{2}$, as the reference material for accelerator-based radiation testing of multi-function composites that are currently being developed. A detailed discussion of the shielding properties of polyethylene under a variety of relevant experimental conditions is presented, along with Monte Carlo simulations of the experiments and other Monte Carlo calculations in which the entire GCR flux is simulated. The Monte Carlo results are compared to the accelerator data and we assess the usefulness of $1 \mathrm{GeV} / \mathrm{amu}{ }^{56} \mathrm{Fe}$ as a proxy for GCR heavy ions. We conclude that additional accelerator-based measurements with higher beam energies would be useful.
\end{abstract}

\section{Introduction}

Much of the motivation for this work is derived from the NASA Space Radiation Health Program [1]. Finding effective approaches to shielding against radiation is one of the highest priority issues. The scope of the risks associated with the health, safety and performance of crews exposed to ionizing radiation during space flight have been identified previously [2]. Here, we focus on the particular importance of polyethylene as a reference material for laboratory tests of shielding materials. For most of the particles and energies found in the Galactic Cosmic Ray (GCR) spectrum, the effectiveness of a material as a radiation shield generally increases with decreasing atomic number, with hydrogen being the best [3, 4], as described in detail in the accompanying paper [5], in which many materials were tested in a beam of $1 \mathrm{GeV} / \mathrm{amu}{ }^{56} \mathrm{Fe}$ ions at the Brookhaven National Laboratory's Alternating Gradient Synchrotron (AGS). Since hydrogen is highly effective, one would expect polyethylene, $\mathrm{CH}_{2}$, with two hydrogen atoms and one carbon atom per molecule, to also be an effective shielding material, and in fact blocks of it 
have been added to the crew sleeping quarters on the International Space Station to provide radiation protection [6,7]. Furthermore, polyethylene is readily available, non-toxic, and chemically stable under typical conditions, making it a convenient reference material for shielding tests at heavy-ion accelerators. NASA materials scientists are developing new composites as they seek to create materials that have both high hydrogen content and sufficient tensile strength to serve as structural members in spacecraft. For each target supplied to us for testing in heavy-ion beams, a $\mathrm{CH}_{2}$ target with equal areal density was also supplied, and the $\mathrm{CH}_{2}$ results were used as the standard to which the other materials were compared. As we will show, these comparisons appear to be a reasonable approach for judging the effectiveness of materials against high-energy beams (600 MeV/amu and higher), but perhaps not meaningful at the lower energies.

Hydrogen is of particular interest for studies of GCR propagation through interstellar space, and also as a component of spacecraft shielding. Accordingly, we have measured nuclear cross sections for many beam ions and energies incident on hydrogen targets [8-12]. The fabrication and use of liquid hydrogen targets are problematic, so as a practical matter the required data are most easily obtained by making cross section measurements for both polyethylene and carbon targets. In the data analysis, carbon-target cross sections are subtracted from the polyethylene results, yielding hydrogen-target cross sections. As a byproduct of these efforts, there are a considerable number of ion/energy combinations for which we have obtained data using the same $2.83 \mathrm{~g} \mathrm{~cm}^{-2}$ polyethylene target. By using the same target for a wide variety of beams, we are able to describe for the first time the systematic dependencies of shield performance on ion species and energy for GCR-like particles. Taken as a whole, our experimental data can be considered a simulation of highly-relevant portions of the GCR spectrum. In addition, we have simulated the shielding properties of polyethylene against the full GCR spectrum using a Monte Carlo computer code, described below.

Although we have obtained data for several ions and energies, our focus to date has been on the portion of the energy spectrum below $1 \mathrm{GeV} / \mathrm{amu}$. This is a necessary, but not sufficient, set of measurements. As we will show, data at higher energies are needed to complete our understanding of the shielding properties of polyethylene and other materials. 


\section{Materials and Methods}

\subsection{Experimental Setup}

A particle spectrometer made of a stack of silicon detectors was used for all measurements; our experimental methods [13] and data analysis techniques [8-11]. A typical beamline configuration as used at the Heavy Ion Medical Accelerator in Chiba (HIMAC, located at the National Institute of Radiological Science in Japan) is shown schematically in Figure 1. Most of the data presented here are from experiments performed at HIMAC; the rest are from the Brookhaven AGS and NSRL accelerators. For most of the experiments, a small $\left(50 \mathrm{~mm}^{2}\right.$ active area) silicon detector, referred to as TR, was mounted in the most upstream location to initiate the experimental trigger and to define the beam area. A $3 \mathrm{~mm}$ thick silicon detector, labeled $\mathrm{d} 3 \mathrm{mmU}$ in Figure 1, was placed just downstream of TR and was used in the off-line analysis to resolve the species of the particles emerging from the vacuum line and entering the target based on the deposited energy, $\Delta \mathrm{E}$, in the detector. This detector, and all other $3 \mathrm{~mm}$ thick detectors used here, are lithiumdrifted and have active radii of $1.15 \mathrm{~cm}$. Cuts are made so that our sample consists only of events in which a single ion of the accelerated beam species was recorded in both TR and $\mathrm{d} 3 \mathrm{mmU}$. In a small number of the experiments reported here (those performed at the AGS and NSRL), it is conceivable that some contamination of our spectra arises from beam particles outside the radius of $\mathrm{d} 3 \mathrm{mmU}$; however, as discussed in detail in the Appendix, we expect this source of contamination to be negligible.

Polyethylene targets were placed downstream of $\mathrm{d} 3 \mathrm{mmU}$. Downstream of the target position, two $3 \mathrm{~mm}$ thick silicon detectors record fragments and surviving primary ions emerging from the target. Immediately downstream of this pair was a pair of silicon position-sensitive detectors (PSDs). These have active radii of 2.0 centimeters and thicknesses of 800-1050 $\mu \mathrm{m}$. The PSDs provide spatial resolution and species identification, though typically with less $\Delta \mathrm{E}$ resolution than the $3 \mathrm{~mm}$ detectors. Because the first pair of $3 \mathrm{~mm}$ detectors and the PSD pair were placed near the target exit, they subtend relatively large angles of acceptance, which is appropriate for our measurements of charge-changing cross sections. An unavoidable consequence of this close placement, however, is the loss of apparent resolution in the spectra below about half the beam charge $\left(Z_{\text {beam }} / 2\right)$. This effect is not due to any degradation of the detector response, but to the 
presence, in some events, of multiple light fragments exiting the target and striking the detectors simultaneously.

A pair of $5 \mathrm{~mm}$ thick silicon detectors $(\mathrm{d} 5 \mathrm{~mm} 1$ and $\mathrm{d} 5 \mathrm{~mm} 2)$ and a second pair of $3 \mathrm{~mm}$ thick detectors ( $\mathrm{d} 3 \mathrm{~mm} 3$ and $\mathrm{d} 3 \mathrm{~mm} 4)$ were mounted downstream of the second PSD to provide highresolution particle identification of ions at small acceptances. The $5 \mathrm{~mm}$ detectors have active radii of about $1.95 \mathrm{~cm}$, nearly double that of the $3 \mathrm{~mm}$ detectors. These far-downstream detectors were typically placed about 1 to 1.5 meters behind the target. Since these detectors subtend smaller acceptance angles and are typically hit by a lower multiplicity of fragments on a given event, they can be used to identify the most forward-going fragment(s) in these events. See Ref. [5] for a comparison of the spectra seen with detectors having large and small acceptances.

The data reported here were obtained with seven beam energies. We refer to the beam energy at extraction from the accelerator; actual energies at the target were slightly lower due to energy lost in the detectors upstream of the target. The extraction energies are 290, 400,600,650, 800, 1000 , and $1200 \mathrm{MeV} / \mathrm{amu}$. In the interests of simplicity, the $650 \mathrm{MeV} / \mathrm{amu}$ data for ${ }^{40} \mathrm{Ar}$ are grouped with the $600 \mathrm{MeV} / \mathrm{amu}$ data in the analysis; similarly, the $1200 \mathrm{MeV} / \mathrm{amu}{ }^{28} \mathrm{Si}$ data are grouped with other data at $1000 \mathrm{MeV} / \mathrm{amu}$. A complete list of ion species and energies used in the various experiments is shown in Table 1.

\subsection{Data Analysis}

The energy deposited in the silicon can be accurately calculated using the Bethe-Bloch formula for stopping power $(\mathrm{dE} / \mathrm{dx})$, which is proportional to the square of ion charge [14]. Since forward-going fragments tend to have velocities very near that of the incident ion, the $\Delta \mathrm{E}$ signal is proportional to the sum of the square of the charges, $\mathrm{Z}_{\mathrm{i}}$, of the fragments that hit a given detector. For each event, the effective measured charge $\left(Z_{\text {eff }}\right)$ in any detector is defined as:

$$
Z_{e f f}=\sqrt{\sum_{i} Z_{i}^{2}} \propto \sqrt{\Delta E}
$$

Eqn.1

Histograms of the effective charge were made and the numbers of each species were determined by counting the peaks, each of which is delimited by a valley on either side. As determined by 
Eqn.1, if a heavy 'leading' fragment is present, its charge dominates the energy deposition for multiple hits in the detector during a single trigger. For example, simultaneous measurement of one carbon ion and one proton would produce a $Z_{\text {eff }}$ of 6.08 , whereas measurement of a carbon and helium would yield a $Z_{\text {eff }}$ of 6.32 . Both of these scenarios would contribute to the width of the carbon peak, and in our methodology would be counted as a single carbon fragment.

Pulse-height information from TR and $\mathrm{d} 3 \mathrm{mmU}$ was used to select events in which only a single, well-measured primary ion was incident on the target. After selection cuts, histograms of charge were generated and the fluence in each charge peak was summed and corrected for background as determined from target-out data. Small (on the order of $5 \%$ or less) but slightly modeldependent correction factors were applied, as a function of species, to compensate for the small distortion in the measured spectrum that arises from the greater probability of interaction for heavier ion species. The correction factors applied to the measured data are discussed in [8-10].

\subsection{Metrics}

The $\Delta \mathrm{E}$ signal in each detector was converted to LET in off-line analysis using a multiplicative constant (scale factor). For a given beam ion and energy, the peak $\Delta \mathrm{E}$ from the primary ion in target-out data was scaled to the LET of the incident ion in order to establish the scale factor ${ }^{1}$. Primaries and fragments were counted and the number of particles, $N(Z)$, was recorded as a function of charge. LET was assigned to a given fragment charge based on $\mathrm{Z}^{2}$ scaling relative to the peak of the primary distribution. Events with multiple fragments are, in the large majority of cases, counted as having the charge of the leading (highest-charge) fragment.

Since there is no generally agreed-upon method of quantifying shielding effectiveness, a simple analysis using the first and second moments of the LET distributions, implemented in previous analyses [6, 7], is used again here. These values, the track- and dose-averaged LET, are closely related to energy deposition and, therefore, to absorbed dose. LET, the energy loss per unit track length ( $\mathrm{dE} / \mathrm{dx}$, usually given assuming the medium being traversed is water) is defined in terms of a single projectile. In our experiments, multiple particles may be recorded during a single

\footnotetext{
${ }^{1}$ The LET of the primary beam is calculated using the energy of the beam as determined by the accelerator.
} 
trigger, especially in the detectors just downstream of the target, so it is not possible to calculate a true track-averaged LET. We therefore use the terminology 'event-averaged' LET to denote the first moment of the spectra under experimental conditions. The event-averaged LET, $L_{\text {event }}$, is mathematically similar, but not identical to, track-averaged LET as given by the relations:

$$
L_{\text {track }}=\frac{\int \frac{d \phi}{d L} L d L}{\int \frac{d \phi}{d L} d L} \quad \text { and } \quad L_{\text {event }}=\frac{1}{N_{e v}} \sum_{Z} L(Z) N(Z) \quad \text { Eqn.2 }
$$

where:

$$
\begin{aligned}
& L_{\text {track }}=\text { track-averaged LET; } \\
& L_{\text {event }}=\text { event-averaged LET; } \\
& L(Z)=\text { average } \mathrm{LET} \text { in } \mathrm{keV} / \mu \mathrm{m} \text { of the particle with charge } \mathrm{Z} ; \\
& N(\mathrm{Z})=\text { number of particles with charge } \mathrm{Z} \text {; and } \\
& N_{\text {ev }}=\text { total number of events in the data sample }
\end{aligned}
$$

We identify the fluence, $\phi(Z)$, which has units of particles per $\mathrm{cm}^{2}$, with the dimensionless quantity $N(Z)$. Though not rigorously correct, as our detectors are greater than $1 \mathrm{~cm}^{2}$ in area, this is nonetheless a good approximation since we measure surviving primaries and fragments that go in the direction of the incident primary with little or no deflection. More importantly, the definitions in Eqn. 2 show the difference between the "event-averaged" quantity and the true track average: for $L_{\text {event }}$, we divide by the number of events recorded and not by the sum of the number of particles present, because the latter quantity is not measured in our experimental setup. Thus $L_{\text {event }}$ is proportional to the dose per incident beam ion behind shielding, and is only equal to $L_{\text {track }}$ when there is no fragment in the event (i.e., the primary survives traversal of the target). In all other cases, where there are - in view of charge conservation - at least two fragments produced, the two average quantities diverge in meaning, and the track average can be much smaller than the event average if there are many light particles present.

In contrast, the dose-averaged LET, $L_{\text {dose }}$ (defined in eqn. 3 below), and the average quality factor are both dominated by large energy deposition events. Low-LET particles have a small effect on these quantities. We retain the usual terminology, however it is important to note that because the denominator depends on $L_{\text {event }}, L_{\text {dose }}$ as operationally defined here differs in meaning from its formal definition. 


$$
L_{\text {Dose }}=\frac{\int L^{2}(Z) \phi(Z) d L}{\int L(Z) \phi(Z) d L}=\frac{1}{L_{\text {event }}} \sum_{Z} L^{2}(Z) N(Z)
$$

Radiation dose is the energy deposited per unit mass. In the planar geometry of our experiments, dose is proportional to the integral of the product of fluence $(\phi)$ and LET, integrated over the full LET spectrum [15]. With multiplication by the appropriate constants, assuming water as the material of interest, the LET spectrum converted from $\Delta \mathrm{E}$ in silicon can be integrated to obtain total dose. We measure the number of particles as a function of species, $N(Z)$, so dose can be expressed as a sum over all charges:

$$
D=\frac{1.602}{N_{e v}} \sum_{Z} L(Z) N(Z)=1.602 \cdot L_{e v e n t}
$$

where $D$ is the dose in nGy and 1.602 is the conversion factor into SI units.

In radiation biology, differences in the relative biological effectiveness (RBE) of different ions are attributed, in part, to differences in the LET of the radiation [16]. For radiation protection, radiation weighting factors, $\mathrm{w}_{\mathrm{r}}$, have been defined to account for differences in RBE between different types of radiation [16]. For mixed fields and radiations without a defined $\mathrm{w}_{\mathrm{r}}$, the ICRP gives a formula for calculating a radiation quality factor, $Q(L)$, which is a function of the LET of the incident particle [16]. The average quality factor in a mixed field, $Q_{a v}$, is defined as:

$$
Q_{a v}=\frac{1}{D} \int Q(L) D(L) d L=\frac{1}{L_{\text {event }}} \sum_{Z} Q(L(Z)) L(Z) N(Z)
$$

where $Q(L)$ is the quality factor for a given value of LET, and $D(L) d L$ is the absorbed dose at LET between $L$ and $L+d L$. The far-right hand side of the equation represents the translation of the formally-correct integral into a sum over a charge histogram, which is the spectrum we generate with our data. Multiplying the absorbed dose by $\bar{Q}$ gives the dose equivalent $H$ in Sieverts (Sv). Using epidemiological data, $H$ can be related to the excess cancer risk arising from the mixed field exposure. The result can be expressed as:

$$
H=D \bar{Q}=D \times \frac{1}{D} \int Q(L) D(L) d L=1.602 \sum Q(L(Z)) L(Z) N(Z) \quad \text { Eqn.6 }
$$


where $H$ is the dose equivalent in nSv.

In [5], we devised an extremely simple method of comparison between experiments with different target depths, in which we compute the fractional dose reduction and divide by the areal density of the target. The resulting quantity, $\delta \mathrm{D}_{\mathrm{n}}$, is (for high-energy beams) roughly independent

of target depth over the first few $\mathrm{g} \mathrm{cm}^{-2}$. It was also shown in [5] that a better method is to measure dose vs. depth curves and extrapolate them to zero depth. Here, we present data obtained with a single target, and for the most part we lack dose vs. depth information for the various beams and energies. Accordingly, we use $\delta D_{n}$ as defined in [5]:

$$
\delta \mathrm{D}_{\mathrm{n}}=\frac{1}{\rho x}\left(1-\frac{L_{\text {event }}}{L_{\text {incident }}}\right)
$$

where $\rho$ is the density of the target, $x$ is its depth, and $L_{\text {incident }}$ is the LET of the primary beam ion at the target entrance.

\subsection{Uncertainties}

As discussed in the accompanying article [5], the uncertainties associated with the measurements presented here are dominantly systematic in nature and arise from the unavoidably arbitrary nature of the graphical cuts used in the data analysis. In [5], we reported results that in several instances included measurements of exactly the same target in more than one experiment. The scatter in the results for a given target, as measured by the standard deviation, was found to be typically about $4 \%$ of the mean, and on that basis we have assigned an uncertainty of $\pm 5 \%$ to all measurements presented here. In the following, the uncertainties are in most cases comparable to, or smaller than, the plotting symbols used. When we present the results of Monte Carlo calculations, we do not show statistical uncertainties since they are negligible. The dominant uncertainties in such calculations are systematic, arising from the many approximations used, as discussed in more detail below. 


\section{Results}

As discussed in previous papers [17,18], the Bragg curve (i.e., dose vs. depth) for a heavy ion beam in a particular material is strongly dependent on the beam ion and its energy. It also depends strongly on the material being traversed [5]. For a given material and beam ion, a change in energy can produce striking differences in the Bragg curve, as was shown in [18], where curves for ${ }^{56} \mathrm{Fe}$ at $600 \mathrm{MeV} / \mathrm{amu}$ and $1 \mathrm{GeV} / \mathrm{amu}$ were compared. Similarly, for a given target and beam energy (per nucleon), a change in the beam species can make a dramatic difference, as the present data show.

\subsection{Fragmentation Physics Considerations}

Both of the important physical processes in heavy ion transport - ionization energy loss and nuclear fragmentation - occur at higher rates in hydrogen than other materials. (See the discussion in [7].) Therefore, per unit mass of shielding, hydrogen stops more of the incident low-energy particles and also causes more fragmentation of high-energy heavy ions than do other materials. It is therefore expected on theoretical grounds that hydrogenous materials should make efficient shields against the GCR, and the experimental results presented in [5] for highenergy ${ }^{56} \mathrm{Fe}$ support this conclusion. The point is further illustrated in Figure 2, which shows a comparison of the fragmentation of $1 \mathrm{GeV} / \mathrm{amu}{ }^{56} \mathrm{Fe}$ for equal areal densities of polyethylene and aluminum. The number of fragments produced per incident beam ion is much higher behind the $\mathrm{CH}_{2}$ shield than for aluminum. Thus $\mathrm{CH}_{2}$ gives a greater dose reduction per $\mathrm{g} \mathrm{cm}^{-2}$ of shield mass than aluminum for this projectile. It is also apparent from Figure 2 that nuclear interactions of $\mathrm{Fe}$ in aluminum are more likely to produce a very light (charge 1 or 2 ) leading fragment than are interactions in $\mathrm{CH}_{2}$. This can be understood in a simple picture of overlapping spheres, which suggests that even in a head-on (central) collision of ${ }^{56} \mathrm{Fe}$ with a much lighter nucleus such as ${ }^{12} \mathrm{C}$

or ${ }^{1} \mathrm{H}$, it is likely that one or more large remnants of the projectile will survive. This gives heavier target nuclei an advantage, but it does not come close to overcoming the advantage that light nuclei have in producing more fragmentation reactions per unit mass. 


\subsection{Depth vs. Dose}

Both here and in [5], we present results in terms of $\delta \mathrm{D}_{\mathrm{n}}$, defined above. The validity of this approach for thick targets is questionable since the Bragg curves of the various beams are not necessarily linear with depth (though in some cases they are). To illustrate the point, we show in Figure 3 the relationship between dose reduction and target depth for $1 \mathrm{GeV} / \mathrm{amu}{ }^{16} \mathrm{O}$ on $\mathrm{CH}_{2}$. The range of this beam in $\mathrm{CH}_{2}$ is approximately $75 \mathrm{~g} \mathrm{~cm}^{-2}$. For target depths up to about $5 \mathrm{~g} \mathrm{~cm}^{-2}$, $\delta \mathrm{D}_{\mathrm{n}}$ is approximately independent of depth (i.e., the Bragg curve is linear with depth), as indicated by the fact that the measured points lie close to the dashed line. As target depth increases, $\delta \mathrm{D}_{\mathrm{n}}$ deviates from linearity for two reasons: first, the LET of the projectile and higher$\mathrm{Z}$ fragments begins to increase more rapidly; second, as depth increases, the number of beam ions available to fragment into lighter ions is depleted by fragmentation events at shallower points in the target. This behavior is typical in high-energy beams and is essentially the same behavior that was described in [5] as the flattening of the $1 \mathrm{GeV} / \mathrm{amu}{ }^{56} \mathrm{Fe}$ Bragg curve with increasing polyethylene depth.

In [5], for data obtained with a $1 \mathrm{GeV} / \mathrm{amu}{ }^{56} \mathrm{Fe}$ beam, $\delta \mathrm{D}_{\mathrm{n}}$ as a function of depth was found to be well-approximated by an exponential, with the curves for lighter materials falling by about 2$3 \%$ per $\mathrm{g} \mathrm{cm}^{-2}$, and those for heavier materials being flatter. Thus for measurements using highenergy beams at depths as great as $5 \mathrm{~g} \mathrm{~cm}^{-2}$, we expect the $\delta \mathrm{D}_{\mathrm{n}}$ results to be within $10-15 \%$ of the results one would obtain with vanishingly thin targets, making the interpretation of results straightforward. However, for the low energy ions that comprise part of the present study, energy loss vs. depth rises rapidly, even in the first few $\mathrm{g} \mathrm{cm}^{-2}$, and the behavior of $\delta \mathrm{D}_{\mathrm{n}}$ with target depth observed at high energy should not be expected to hold, even approximately.

\subsection{Shielding Results with a $2.83 \mathrm{~g} \mathrm{~cm}^{-2} \mathrm{CH}_{2}$ Target}

Figure 4 shows the most important result of the current study: dose reduction behind a single $\mathrm{CH}_{2}$ target (depth $2.83 \mathrm{~g} \mathrm{~cm}^{-2}$ ) for nineteen different beam ion/energy combinations. Large variations are seen, demonstrating the projectile and velocity dependence of shielding effectiveness. For the range of energies studied, larger dose reductions result from the higher energy, heavier projectiles. At $290 \mathrm{MeV} / \mathrm{amu}$, there is close to no shielding effect for $\mathrm{C}$ and $\mathrm{N}$ 
beams, and a slight increase in $L_{\text {event }}$ is seen for $\mathrm{Ne}$, shown as a negative dose reduction. At 400 $\mathrm{MeV} / \mathrm{amu}$, the shield reduces the dose for projectiles from $\mathrm{C}$ to $\mathrm{Ne}$ (charge 10), but as $Z_{\text {beam }}$ increases, the curve turns over so that for $\mathrm{Si}$, the shield has little effect, and for Ar, the shield actually increases the dose per incident ion. At energies of 600,800 , and $1000 \mathrm{MeV} / \mathrm{amu}$, the dose reductions are more substantial, 2 to $5 \%$ per $\mathrm{g} \mathrm{cm}^{-2}$, and appear to be almost independent of the ion species from $\mathrm{O}$ to $\mathrm{Fe}$. The measured dose reductions increase steadily with increasing beam energy in this range.

A summary of the data in Figure 4 is given in Table 2. Ions with ranges much greater than the shielding depth show a positive dose reduction, since there is only minimal change in the LET of primaries that survive transport through the shield, and the effects of fragmentation dominate. In contrast, for the relatively light ions measured at $290 \mathrm{MeV} / \mathrm{amu}, L_{\text {event }}$ is unchanged to within $\pm 0.5 \%$ from the LET of the incident ion. In these cases, the increase in the LET of the surviving projectiles almost precisely balances the reduction in average LET caused by fragmentation. The dose-averaged LET remains within $12 \%$ of the LET of the incident ion in all cases.

Radiological properties of the data presented in Table 2 are given in Table 3. Absorbed dose (Eqn. 4) follows the trend in event-averaged LET since it differs only by a conversion factor. Some projectile/energy combinations show an increase in dose-averaged LET (Eqn. 3) even though the $L_{\text {event }}$ is lower than the LET of the incident ion. For these particles, $Q_{a v}$ behind the shield is also higher than the $Q$ of the incident ion. Changes in $Q_{a v}$ (increases are shown as negative numbers in the table) are significant at low beam energy. For instance, in the case of $290 \mathrm{MeV} / \mathrm{amu}{ }^{12} \mathrm{C}, L_{\text {event }}$ is equal to the beam LET, but the change in $Q_{a v}$ leads to a $15 \%$ increase in dose equivalent per incident beam ion.

The behavior of the average quality factors after the shield is complex. Biological experiments show that though shielding decreases the biological effectiveness of the primary beam, neither $L_{\text {event }}$ nor $L_{\text {dose }}$ correlates well with the effectiveness of the mixed charged particle field generated by nuclear fragmentation [19]; it appears likely that no single variable can adequately describe the relation between the composition of a mixed field and its biological effectiveness. From the perspective of risk management for spaceflight, the use of quality factor (with all its inherent uncertainty) to incorporate these complexities and yield a single value that is a meaningful 
indicator of risk is problematic. However, this approach is required by current regulatory mandates. Here, we do not attempt to assess the utility of the present risk assessment methodology; rather, we demonstrate the consequences - some of which are not obvious - of the present definition of $\mathrm{Q}(\mathrm{L})$ vis a vis the changes in mixed fields arising from traversal of polyethylene shielding. We define the change in average quality to be

$$
\Delta Q=\left(Q_{i n}-Q_{a v}\right) / Q_{i n} \quad \text { Eqn. } 8
$$

where $Q_{\text {in }}$ refers to the $Q$ of the incident beam before the target.

A few trends can be seen in the data: (1) for a given beam species, as energy increases, $\Delta Q$ becomes more positive, for beams from $\mathrm{C}$ to $\mathrm{Si}$; (2) for beam energies of $600 \mathrm{MeV} / \mathrm{amu}$ and above, the $\Delta Q$ are all positive, except for ${ }^{16} \mathrm{O}$, for which $\Delta Q \approx 0$; (3) for 290 and $400 \mathrm{MeV} / \mathrm{amu}$, the $\Delta Q$ are all negative (i.e., $Q_{a v}$ after the shield is greater than $Q$ of the incident beam ion), with the exception of $400 \mathrm{MeV} / \mathrm{amu}$ Ar.

The largest decreases in $Q$ (i.e., most positive values of $\Delta Q$ ) are for $\mathrm{Ti}$ at $1 \mathrm{GeV} / \mathrm{amu}$ and $\mathrm{Ar}$ at $400 \mathrm{MeV} / \mathrm{amu}$. In Table 2, we see that for these two beams, the LET of the incident primary ion is quite close to $100 \mathrm{keV} / \mu \mathrm{m}$, the inflection point of the quality factor curve, and the point at which the first derivative, $\mathrm{dQ} / \mathrm{dL}$, is discontinuous. Below $100 \mathrm{keV} / \mu \mathrm{m}, \mathrm{dQ} / \mathrm{dL}$ is constant, with a value of $0.32(\mathrm{keV} / \mu \mathrm{m})^{-1}$; above $100 \mathrm{keV} / \mu \mathrm{m}, \mathrm{dQ} / \mathrm{dL}=-150 \mathrm{~L}^{-3 / 2}$, and attains its most negative value of $-0.15(\mathrm{keV} / \mu \mathrm{m})^{-1}$ precisely at $100 \mathrm{keV} / \mu \mathrm{m}$. We believe the behavior of $\Delta Q$ for these two beams is related to the non-physical behavior of $\mathrm{dQ} / \mathrm{dL}$ in this region. In both cases, the LET of the primary beam ions that survive the target shift from being below the peak of $\mathrm{Q}$ to being above the peak, and virtually all fragments have a lower Q than did the incident primaries. The Monte Carlo results presented below reproduce the effect fairly well.

In most of the data, $Q_{a v}$ is within a few percent of the $Q$ of surviving primaries. This is not surprising since $Q_{a v}$ is a dose-weighted quantity, and because the majority of primaries (67\% to $89 \%$ depending on the beam) survive the target, they are invariably the dominant component of the dose and dose equivalent behind the target. 


\subsection{Monte Carlo Simulations of Experiments}

\subsubsection{One-Dimensional Transport Model}

Monte Carlo methods are employed in a transport code [20] written at LBNL that joins the NUCFRG2 [21] cross section database with detailed energy-loss calculations using the BetheBloch equation. The code is called BBFRAG. All nineteen of the data sets that appear in Tables 2 and 3 and related figures were simulated. In addition, the transport of particles through thick absorbers was simulated for two cases in order to illustrate the competing effects of fragmentation and ionization energy loss in the way dose changes as a function of depth in water or tissue. The Monte Carlo code does not include straggling in its energy loss calculations, so when it is used to produce a Bragg curve, it gives sharper peaks that would be obtained in a real experiment. Further, when a nuclear interaction occurs in the simulation, only one fragment - the "leading" fragment - is produced and followed. This keeps the code simple and is a reasonable approximation for the majority of cases, in which the interaction is peripheral, few nucleons are removed from the projectile, and the fragment multiplicity is low. However this approximation bears little resemblance to reality for central collisions, in which the multiplicity of non-leading fragments can be large. Two other readily-available codes [22, 23] could have been used to model experimental data, but use of these codes on a data set this large would be problematic. One (PHITS) requires long computational times, while HZETRN must be recompiled to change targets. BBFRAG was employed here for its computational speed and adaptability, giving results comparable to HZETRN since important parts of the codes are identical (both use the BadhwarO'Neill model to produce the GCR spectrum, and both use NUCFRG2 cross sections for transport).

Figure 5 is a scatter plot of the $\Delta Q$ and $\delta \mathrm{D}_{\mathrm{n}}$ values obtained in the data (abcissa) and in the Monte Carlo (ordinate). Points with perfect agreement between data and Monte Carlo would fall on the $45^{\circ}$ line. The $\delta \mathrm{D}_{\mathrm{n}}$ points cluster near the line, tending to fall slightly to the left of the line. Only one point ( $\mathrm{Ar}$ at $400 \mathrm{MeV} / \mathrm{amu}$ ) is far from the line. The $\Delta Q$ points with measured values greater than zero also cluster near the $45^{\circ}$ line, but to the right of the line. In the cases where the magnitude of $\Delta Q$ is relatively large, and its sign negative, the points scatter more, indicating less agreement between data and Monte Carlo. Nonetheless, on the whole, the Monte Carlo 
reproduces the trends seen in the data reasonably well, with some systematic differences that bear examination.

In Figure 5, in almost all cases, $\delta \mathrm{D}_{\mathrm{n}}$ in the Monte Carlo is more positive than in the data, with the reverse being true for $\Delta Q$. Both these trends result from the overly simple fragmentation model implemented in the Monte Carlo, in which only the leading fragment is followed. In the data, non-leading fragments contribute to dose. The absence of non-leading fragments in the Monte Carlo leads to an overestimate of dose reduction because the Monte Carlo $L_{\text {event }}$ tends to be smaller than in the data. This also affects $\Delta Q$ in the Monte Carlo in a slightly more subtle way. In eqn. 8 above, for a given beam, $Q_{i n}$ will be the same for data and Monte Carlo - it is simply $Q(L)$ calculated for L of the primary beam. Therefore, all differences between data and Monte Carlo in $\Delta Q$ will be due to differences in $Q_{a v}$, which is inversely proportional to $L_{\text {event }}$ (see eqn. 5 above). We expect that the numerator of eqn. 5 is approximately correct in the Monte Carlo since it is typically most heavily weighted by the surviving primaries, which are accurately simulated. However the Monte Carlo underestimates $L_{\text {event }}$, and therefore will tend to overestimate $Q_{a v}$, which in turn yields a smaller $\Delta Q$ than in the data.

To illustrate the ion and energy dependencies of the effects of energy loss and fragmentation, we have used BBFRAG to calculate Bragg curves for two substantially different cases. In Figure 6a, we show LET vs. depth ${ }^{2}$ in $\mathrm{CH}_{2}$ of $1 \mathrm{GeV} / \mathrm{amu}{ }^{56} \mathrm{Fe}$, with the upper set of points showing the LET of the primary at a given depth, the middle set of points the dose-averaged LET, and the lowest set the event-averaged LET. The event-averaged LET behind the shield is lower than the LET of the primary for all depths, and a dose is deposited beyond the Bragg peak due to fragments with longer ranges than the primary. The curve for dose-averaged LET falls below but stays relatively close to the LET of the primary and remains quite high beyond the Bragg peak. The dose just past the Bragg peak is due to the contribution of heavy charged fragments produced upstream. These ions have greater ranges slightly greater than that of the primary, but are slowed by the thick target and stop just beyond the depth at which primaries stop. As these fragments approach the end of their ranges, their LET rises and they exert a strong pull on the

\footnotetext{
${ }^{2}$ In the Monte Carlo, the density of polyethylene is taken to be $0.90 \mathrm{~g} \mathrm{~cm}^{-3}$. Thus the depth values given in cm can be trivially converted to units of $\mathrm{g} \mathrm{cm}^{-2}$ by applying this factor.
} 
dose-averaged LET; they have a much weaker pull on the event-averaged LET, which decreases steadily beyond the Bragg peak. We note that the event-averaged curve shown here for $\mathrm{CH}_{2}$ is very similar to data shown in [24] for $\mathrm{H}_{2} \mathrm{O}$, with the $1 \mathrm{GeV} / \mathrm{amu}{ }^{56} \mathrm{Fe}$ beam at the Brookhaven AGS.

To show a contrasting case, we have also modeled a beam for which the event-averaged dose per particle increases behind a shield, $290 \mathrm{MeV} / \mathrm{amu}{ }^{28} \mathrm{Si}$. In this case, both the event- and doseaveraged LET at depths are higher than the LET of the unshielded beam (zero depth) as shown in Figure $6 \mathrm{~b}$. The increase with depth continues until the point at which the projectile stops. The increased LET of the projectile from ionization energy loss dominates over the effects of fragmentation, for all depths up to the range of the primary. One indicator of this is that the doseaveraged LET points fall almost on top of the points calculated for the primary ${ }^{28} \mathrm{Si}$ only. Even the event-averaged LET follows a similar trend to the other two curves. Beyond the Bragg peak, we again see that fragments produce a dose at depths well beyond the range of the incident beam ions.

\subsubsection{Neutron Dose Using PHITS}

The dose from neutrons produced by $1 \mathrm{GeV} / \mathrm{amu}{ }^{56} \mathrm{Fe}$ incident on $\mathrm{CH}_{2}$ was calculated using the PHITS Monte Carlo code [25]. The dose equivalent vs. depth is shown in Figure 7; it rises sharply at shallow depths but flattens out as the depth nears the range of the Fe and stops. The neutron dose beyond $20 \mathrm{~cm}$ is nearly constant, which is not an obvious result, since neutrons do not lose energy through ionization.

At depths much greater than the range of the incoming Fe, heavy charged fragments begin to range out, and their contribution to total dose and dose equivalent decreases. At very great depths, the charged particle dose is due entirely to high energy singly-charged particles (protons, muons, and charged pions) and helium ions, all of which have very long ranges in $\mathrm{CH}_{2}(3.4$ meters for a $1 \mathrm{GeV}$ proton). Although the dose from neutrons is much less than that from the charged particles along the beam axis for modest shielding depths, the neutron component becomes significant at large depths, or when one looks at relatively large angles with respect to the incident primary particle. Neutrons must therefore be accounted for when planning for deeply-buried lunar and planetary habitats. 


\subsection{Monte Carlo Simulations of the GCR Environment}

\subsubsection{Shielding By The $2.83 \mathrm{~g} \mathrm{~cm}^{-2}$ Polyethylene Target}

The BBFRAG Monte Carlo code was modified from its original form, in which accelerator experiments with a single incident ion and energy were simulated, to simulate an incident particle flux generated using a parameterization of the Badhwar-O'Neill GCR model [26]. (We refer to this model as "B-ON" in the following.) All the results in this section were obtained with this code, which allows for a wide variety of possible target materials. Here, the $2.83 \mathrm{~g} \mathrm{~cm}^{-2} \mathrm{CH}_{2}$ target was simulated.

The GCR flux predicted by B-ON consists of about $86 \%$ protons, $12 \%$ He ions, and $2 \%$ heavier ions. The model has a single free parameter, the solar deceleration parameter $\Phi$. Choosing a $\Phi$ value fixes the energy distributions. We used a relatively large value of $\Phi$, corresponding to the 2002-2003 period, just after solar maximum. This selects a relatively "harder" spectra (larger fraction of the flux at high energy) than would exist at a time frame closer to solar minimum. For the chosen time frame, the proton energy distribution has a mean of about $900 \mathrm{MeV}$, while distributions for other nuclei (with $\mathrm{Z} / \mathrm{A} \approx 1 / 2$ ) have means of 575-600 MeV/amu. Protons and He at high energies will barely be affected by the thin $\mathrm{CH}_{2}$ shield used in the simulation, so to first order one might expect little or no shielding effect, since some $98 \%$ of the incident particles will be essentially unaffected. However, the BBFRAG simulation predicts a $\delta D_{n}$ of $2.3 \%\left(\mathrm{~g} \mathrm{~cm}^{-2}\right)^{-1}$. Considering how little of the GCR flux consists of heavy ions, and the relatively modest dose reductions (or even dose increases) seen in Figure 4, this is a remarkable result.

We can understand the relatively large dose reduction with a straightforward argument. When the fluxes predicted by the B-ON model are weighted by $Z^{2}$ to get an estimate of dose contributions, protons and He account for about $63 \%$ of the total. To a good approximation, the $2.83 \mathrm{~g} \mathrm{~cm}^{-2} \mathrm{CH}_{2}$ shield has no effect on these particles. The simulation of GCR heavy ions only (charge 3 and greater) yields a dose reduction of $7.0 \%\left(\mathrm{~g} \mathrm{~cm}^{-2}\right)^{-1}$; if we multiply this by the approximately $37 \%$ dose fraction represented by the heavy ions, we arrive at an overall $\delta \mathrm{D}_{\mathrm{n}}$ of $2.6 \%\left(\mathrm{~g} \mathrm{~cm}^{-2}\right)^{-1}$. Thus the surprisingly large $\delta \mathrm{D}_{\mathrm{n}}$ result obtained in the full simulation has a fairly simple explanation. 
The difference between the heavy-ion $\delta \mathrm{D}_{\mathrm{n}}$ of $7 \%\left(\mathrm{~g} \mathrm{~cm}^{-2}\right)^{-1}$ and the largest values measured here, about $5 \%\left(\mathrm{~g} \mathrm{~cm}^{-2}\right)^{-1}$, is apparently due to the higher energies of some GCR ions. We recall that $\delta \mathrm{D}_{\mathrm{n}}$ increases with energy, nearly independent of the ion species, and we note that a considerable portion of the heavy-ion flux is at energies above $1 \mathrm{GeV} / \mathrm{amu}$. (This is especially true near solar maximum.) Therefore we conclude that the dose reduction for the GCR heavy ions as a whole is strongly influenced by the high-energy portion of the spectrum above $1 \mathrm{GeV} / \mathrm{amu}$.

\subsection{2 ${ }^{56} \mathrm{Fe}$ at $1 \mathrm{GeV} / \mathrm{amu}$ as a GCR Proxy}

In the companion paper to this one [5], we reported and interpreted results obtained with a $1 \mathrm{GeV} / \mathrm{amu}{ }^{56} \mathrm{Fe}$ beam and a variety of target materials. For $\mathrm{CH}_{2}$, the fit to the $\delta \mathrm{D}_{\mathrm{n}}$ vs. depth curve predicts that the $2.83 \mathrm{~g} \mathrm{~cm}^{-2}$ target would give a $\delta \mathrm{D}_{\mathrm{n}}$ value of $4.7 \%\left(\mathrm{~g} \mathrm{~cm}^{-2}\right)^{-1}$. The preceding discussion suggests that the average for GCR heavy ions as a whole at this depth is about $6 \%\left(\mathrm{~g} \mathrm{~cm}^{-2}\right)^{-1}$, some $30 \%$ higher. Since the values of $\delta \mathrm{D}_{\mathrm{n}}$ in Figure 4 are found to be nearly independent of ion species for charges 8 and higher, we attribute the difference to the portion of the HZE energy spectra above $1 \mathrm{GeV} / \mathrm{amu}$ at the higher energies, assuming that $\delta \mathrm{D}_{\mathrm{n}}$ remains roughly independent of species at even higher energies. We conclude that $1 \mathrm{GeV} / \mathrm{amu}{ }^{56} \mathrm{Fe}$, or any other heavy ion with the same energy per nucleon, is a reasonable proxy for the GCR heavy ions, at least for thin targets. We would expect to find a better match with somewhat higher beam energies, perhaps in the range 3-5 GeV/amu.

\subsubsection{Dose vs. Depth of GCR in Polyethylene}

In Figure 8, we show event-averaged LET and dose-averaged quality factor as functions of depth in polyethylene for the incident GCR flux described above. We note that these two quantities are the main ingredients in dose equivalent ${ }^{3}$. In the accelerator experiments performed to date, even at fairly high energy, a distinct Bragg peak is seen when the target depth equals the range of the incident primary beam ion. This is not the case for the GCR, with its more complicated mix of ions and energies. There is no Bragg peak, and instead both $L_{\text {event }}$ and $Q_{a v}$ are seen to monotonically decrease. For example, at a depth of $10 \mathrm{~cm}$, average LET decreases from $0.57 \mathrm{keV} / \mu \mathrm{m}$ to $0.45 \mathrm{keV} / \mu \mathrm{m}$ (a $21 \%$ decrease) and $Q_{a v}$ decreases from 5.8 to 3.6 , a $38 \%$ decrease.

\footnotetext{
${ }^{3}$ The product of the two is the figure of merit vis a vis dose equivalent.
} 
Taking the product of the two numbers at each depth, the $10 \mathrm{~cm}$ value is about half that of the unshielded GCR. The behavior of the GCR $Q_{a v}$ with depth contrasts with that seen in accelerator experiments. For example, with $1 \mathrm{GeV} / \mathrm{amu}{ }^{56} \mathrm{Fe}$, a depth of $10 \mathrm{~cm}$ of $\mathrm{CH}_{2}$ has little effect on $Q_{a v}$, causing it to decrease only by about $5 \%$. The difference in the two results is largely due to the lower-energy heavy ions in the GCR, many of which stop in modest shielding depths without producing secondaries. Therefore they do not contribute to dose, dose equivalent, or $Q_{a v}$ at depth. This has an especially large impact on the latter two quantities since they are both weighted by LET, and these particles tend to have large values of both $L$ and $Q$ as they enter the shield.

As discussed above, the BBFRAG Monte Carlo produces only one fragment per nuclear interaction, meaning that multiplicity is significantly underestimated, especially that of light fragments. Adding these in would increase the dose (and the event-averaged LET as we have defined it), but would slightly decrease $Q_{a v}$, so that the net effect on dose equivalent of the unaccounted-for light fragments is probably small.

\section{Discussion}

Though the heavy ions account for a very small fraction of the GCR flux, when weighted by their respective LET and quality factors they account for a substantial fraction of the dose equivalent during a mission. The data presented here show that a $2.83 \mathrm{~g} \mathrm{~cm}^{-2}$ polyethylene target provides anywhere from a $5 \%$ reduction in dose per $\mathrm{g} \mathrm{cm}^{-2}$ of depth, to a dose increase of $1.1 \%$ per $\mathrm{g} \mathrm{cm}^{-2}$, depending on the projectile species and energy. At the highest energies measured here, the results appear nearly independent of the beam ion species, while at the lower energies, there is a strong dependence on species. Additional measurements with various ions at energies above $1 \mathrm{GeV} / \mathrm{amu}$ would be useful to expand the range shown in Figure 4. Our relatively simple Monte Carlo program, BBFRAG, reproduces the dose reduction data fairly well.

Data shown both here and in [5] indicate that the normalized dose reduction, $\delta \mathrm{D}_{\mathrm{n}}$, is approximately independent of depth for the first few $\mathrm{g} \mathrm{cm}^{-2}$ of $\mathrm{CH}_{2}$, as long as the range of the projectile is much greater than the depth of the shield. As the shield depth increases, $\delta \mathrm{D}_{\mathrm{n}}$ starts to 
decrease, indicating declining shield performance. In Ref. [5], this decrease was accounted for by fitting exponential curves to the data and extrapolating to zero depth to compare the effectiveness of various materials. Shield effectiveness begins to decrease due to two effects: one, at depth, many primaries have already been fragmented into lighter ions; and, two, the energy lost by the projectile $(\mathrm{dE} / \mathrm{dx})$ increases with depth, and this begins to counter the decrease in average LET caused by fragmentation. For ions with lower velocities, the LET rises sharply at depths that are small compared to the mean free path for a nuclear interaction, and the effects of energy loss outweigh those of fragmentation. Put another way, there is in all cases a competition between the effects of energy loss and fragmentation. For high-energy beams, even heavy ions with high LET, fragmentation dominates and dose decreases with depth. As beam energy decreases, the two effects are more nearly equal, and the effect of shielding (as measured by event-averaged LET) is minimal. At still lower energies, for sufficiently heavy beam ions, energy loss dominates over fragmentation, and the shielding actually increases the dose per incident particle.

A Monte Carlo simulation of the GCR impinging on the $2.83 \mathrm{~g} \mathrm{~cm}^{-2} \mathrm{CH}_{2}$ target predicts a $\delta \mathrm{D}_{\mathrm{n}}$ of $2.3 \%\left(\mathrm{~g} \mathrm{~cm}^{-2}\right)^{-1}$, which we find to be reasonably consistent with a straightforward understanding of the various GCR dose contributions. When only the GCR heavy ions are considered, this result is marginally consistent with the shielding results for $\mathrm{CH}_{2}$ obtained in [5] with a $1 \mathrm{GeV} / \mathrm{amu}{ }^{56} \mathrm{Fe}$ beam. While measurements with a single energy and beam ion do not allow us to precisely predict the shielding effectiveness of a material in the full GCR environment, the data presented here suggest that heavy ions with kinetic energies of $1 \mathrm{GeV} / \mathrm{amu}$ and higher may represent a reasonable proxy for the GCR heavy ions for thin and moderately thick targets. Additional Monte Carlo runs with varying depths of $\mathrm{CH}_{2}$ show a depth-dose relation for the GCR that is unlike those encountered in the accelerator environment.

We have presented a set of shielding data for a thin polyethylene target encompassing a wide variety of beam ions and energies, allowing reference comparisons for other materials tested in these same beams. Additional measurements at higher beam energies, using both thin and thick targets, and possibly deploying counters sensitive to the neutron component of the dose, will be of considerable interest. 


\section{Acknowledgements}

We thank the outstanding operations staff of HIMAC, National Institute of Radiological Sciences, Chiba, Japan, and the NSRL, Brookhaven National Lab, Upton, New York for providing particle beams for the experiments. This work was supported by the National Aeronautics and Space Administration, Marshall Spaceflight Center, under Award No. T5606W, and by the NASA Space Radiation Health Program under Grant No. L143230C, through the U.S. Department of Energy under Contract No. DE-AC03076SF00098.

\section{Appendix - Beam Spot Definition and Contamination}

In a recent paper [27], Aiginger et al. used the FLUKA Monte Carlo program to simulate one of the experiments that is a part of the present study. The issue of track-averaged LET vs. doseaveraged LET was discussed in some detail, and track-averaged LET was described as "hopelessly sensitive to experimental conditions." Here and in Ref. [5], we have made an operational definition of "event-averaged" LET, a quantity that is proportional to the dose per incident beam ion. Event-averaged LET differs significantly from track-averaged LET, which is inaccessible to us experimentally since we do not measure the multiplicities of fragments, deltaelectrons, etc. Regardless of what one believes about the utility of track-averaged LET, it should be pointed out that part of the Monte Carlo study presented in Ref. [27] appears to be based on a misunderstanding of our experimental configuration. The authors simulated our experiment with a broad, $7 \mathrm{~cm}$ diameter beam and it appears that, in creating histograms of energy deposition in silicon detectors, particles were simply scored regardless of the location of the incident beam ion. In reality, for all experiments performed by our group, a small trigger detector $-0.8 \mathrm{~cm}$ diameter in HIMAC experiments, $1.95 \mathrm{~cm}$ or $2.2 \mathrm{~cm}$ at the AGS, depending on the experiment - is used to define the beam, selecting only the central portion. (Regardless of size, the trigger detector is referred to here as "TR.") Also, detectors with areas substantially larger than TR were typically placed downstream of TR but upstream of the target, specifically for the reason that the pulse heights from those detectors tell us whether a second particle was present outside TR. If there was more than one particle present in any of the upstream detectors, the event was rejected. In addition, the timing of the trigger logic is the single largest factor in eliminating this category of contamination, as we will show, but this appears to have been ignored in the Monte Carlo 
simulation. The experimental timing, combined with low beam intensity, makes the detectors placed downstream of the targets largely blind to fragments that may be created by the interactions of primary ions that have radial distances from beam center greater than the radius of TR.

Several factors must combine in order for the readout of detectors downstream of the target to be distorted by a particle from an unrelated incident beam ion at large radius: (1) The beam must be larger in area than TR, which was only true in our AGS and NSRL experiments. (2) Given one beam ion at small radius (so that TR is hit), the second beam ion is at a radius larger than TR, and also outside the area of the other detectors placed upstream of the target, typically including one or more with a $2 \mathrm{~cm}$ radius. (3) The timing is such that two beam ions with geometry as per item (2) arrive coincident within the 5 to $10 \mu \mathrm{sec}$ gating time of our ADC's. (4) The large-radius beam ion interacts in the target, and one or more secondary particles happen to scatter into the solid angle of the detectors downstream of the target. We will examine each of these items in more detail below.

We see no evidence in the AGS data that the contamination described by Aiginger et al. actually occurs. (It cannot occur in the HIMAC experiments because of the small beam.) The key mitigating factor is the very low beam intensity typical of our experiments. Due to the long shaping times used with our thick silicon detectors, and relatively slow readout, about $150 \mu \mathrm{sec}$ per event, we attempt to keep the rate on the trigger detector in the range 500 to 1000 per spill, with typical spill lengths of 0.5 to 1 second. With the $300 \mathrm{~mm}^{2}$ TR, a worst-case 0.5 second spill length, and using the high end of our acceptable rates, 1000 particles per second hitting TR, the instantaneous beam intensity is $667 \mathrm{~cm}^{-2} \mathrm{~s}^{-1}$. A beam uniform across a $7 \mathrm{~cm}$ diameter would have an instantaneous total of $2.6 \times 10^{4}$ particles $\mathrm{s}^{-1}$ contained in the whole area. With an ADC gating time of $8 \mu \mathrm{sec} \mathrm{ADC}$, as is typical, we expect an average occupancy of 0.21 particle over the entire area per randomly-chosen $8 \mu \mathrm{sec}$ sampling period. Using Poisson statistics, which assumes a uniform distribution of beam arrival times over the spill, we find that $9.9 \%$ of the time when there is one ion present, there is at least one additional ion present in the same time window. Assuming spatial uniformity, with one particle inside the radius of TR, it is overwhelmingly likely (96\% probable) that the second particle will be outside the radius of TR. Multiplying the 
0.099 probability based on the timing distribution by the 0.96 probability based on the spatial distribution, we arrive at an estimate of 0.095 for a "bad geometry/bad timing" configuration of the beam particles. However, recall that if the second particle is outside TR but within the radius of one of the other upstream detectors, the event will be rejected in the offline analysis, so this estimate is extremely conservative in that it ignores the larger detectors. We have also used worst-case values for spill length and beam intensity. Thus a 9.5\% estimate of the probability for an event configuration that allows for possible contamination is a large overestimate, and it is greatly reduced by the factors we consider next.

The actual probability for contamination is much smaller than the very conservative $9.5 \%$ "bad geometry/bad timing" probability arrived at above would suggest, because of the thin targets that are typically used. Typical target depths represent $10-15 \%$ of a nuclear interaction length, and rarely exceed $20 \%$. Taking a conservative value of $20 \%$, the probability for a bad geometry/bad timing event with an interaction of the large-radius primary is simply the product of the two probabilities, i.e., approximately $0.095 \times 0.2=0.0019$. This still substantially overstates the problem, because fragments produced at large radius that happen to hit a downstream detector would simply add to the energy deposited by the particle inside the radius of TR, and that (in this scenario) has an $80 \%$ chance of being a primary. Light fragments with charge 1 or 2 , produced at large radius will add a negligible amount of deposited energy to the signal. E.g., for a charge 2 fragment, the fractional $\Delta \mathrm{E}$ contributed is given approximately by $2^{2} /\left(26^{2}+2^{2}\right)$, or $0.6 \%$, well within the detector resolution. Such an event would be indistinguishable from one in which a single beam ion survived traversal of the target. Thus, for large-radius beam ions to contaminate the measurement in an observable way, the particle inside the radius of TR must also interact. Multiplying the $0.19 \%$ probability obtained above by a $20 \%$ interaction probability gives a total of about $0.04 \%$ that any given event contains observable contamination. Again, this is conservative, because in most interactions, a heavy fragment is produced, and its energy loss in the detector would dominate the recorded $\Delta \mathrm{E}$. Only when the particle within the radius of TR produces relatively light fragments will any contamination from other beam particles be noticeable. 
Finally, consider a beam ion at a $2.5 \mathrm{~cm}$ radius; a secondary would have to deflect by about 0.5 $\mathrm{cm}$ in the plane perpendicular to the beam direction in order to hit a PSD ( $2 \mathrm{~cm}$ radius). Given the typical placement of the PSDs about $30 \mathrm{~cm}$ downstream of the target, this corresponds to a small angle, slightly less than $1^{\circ}$. Deflections of this magnitude or larger are common for the lightest fragments, charges 1 and 2 in particular, but rare for the heavy secondaries created in interactions of the $1 \mathrm{GeV} / \mathrm{amu}{ }^{56} \mathrm{Fe}$ beam. (Most interactions produce a heavy secondary.) For example, the calculated standard deviation for the presumed Gaussian angular distribution of ${ }^{40} \mathrm{Ar}$ fragments is about $0.5^{\circ}$; thus about $95 \%$ of such fragments produced at a radius of $2.5 \mathrm{~cm}$ would be too forward-going to scatter into the downstream acceptance. Although the angular distributions of protons and helium fragments are much broader than those of heavy fragments, the fraction of light fragments that actually would scatter into the acceptance of a detector downstream is still relatively small, since the detector subtends only a portion of the (uniform) azimuthal angular distribution of the fragments. As seen from target center, with the PSD $30 \mathrm{~cm}$ downstream, the detector subtends only 0.014 steradian of solid angle and, at its center, occupies only about $25 \%$ of the azimuth. Taking the $0.04 \%$ probability for a bad geometry/bad timing event in which both primaries interact in the target, and factoring in this azimuthal fraction, we arrive at a final estimate of $0.01 \%$ probability of contamination. Even if the multiplicity of light fragments averaged ten per interaction, the probability would still be only $0.1 \%$. We emphasize that almost certainly an overestimate of the problem, since we have made very conservative estimates at every step, and the real probability is probably much smaller. In any case, a probability on the order of $10^{-3}$ is of negligible importance in the present work, and also in the cross section measurements we have reported elsewhere. 


\section{References}

[1] National Aeronautics and Space Administration, Bioastronautics Critical Path Roadmap (BCPR), An Approach to Risk Reduction and Management for Human Space Flight: Extending the Boundaries, JSC 62577 (2004).

[2] W. Schimmerling, F.A. Cucinotta, J.W. Wilson, Radiation Risk and Human Space Exploration, Adv. Space Res. 31 (2003) 27-34.

[3] J. W. Wilson, S. A. Thibeault, F. A. Cucinotta, J. L. Shinn, M. Kim, R. Kiefer and F. F. Badavi, Issues in protection from galactic cosmic rays. Radiat. Environ. Biophys. 34 (1995) $217-$ 222.

[4] C. Zeitlin, S. Guetersloh, L. Heilbronn, J. Miller, Shielding and Fragmentation Studies, Radiat. Prot. Dosim., 116 (2005) 123-124.

[5] C. Zeitlin, S. B. Guetersloh, L. Heilbronn, and J. Miller, Measurements of materials shielding properties with $1 \mathrm{GeV} / \mathrm{nuc}^{56} \mathrm{Fe}$, Nucl. Instr. Meth. in Phys. Res. B (2006) (submitted).

[6] M.R. Shavers, N. Zapp, R.E. Barber, J.W. Wilson, G. Qualls, L. Toupes, S. Ramsey, V. Vinci, G. Smith, F.A. Cucinotta, Implementation of ALARA radiation protection on the ISS through polyethylene shielding augmentation of the Service Module Crew Quarters, Adv. Space Res. (2004) 1333-1337

[7] J. Miller, C. Zeitlin, F.A. Cucinotta, L. Heilbronn, D. Stephens and J.W. Wilson, Benchmark Studies of the Effectiveness of Structural and Internal Materials as Radiation Shielding for the International Space Station, Radiat. Res. 159 (2003) 381-390.

[8] C. Zeitlin, L. Heilbronn, J. Miller, S. E. Rademacher, T. Borak, T. R. Carter, K. A. Frankel, W. Schimmerling and C. E. Stronach, Heavy fragment production cross sections from 1.05 $\mathrm{GeV} /$ nucleon ${ }^{56} \mathrm{Fe}$ in $\mathrm{C}, \mathrm{Al}, \mathrm{Cu}, \mathrm{Pb}$, and $\mathrm{CH}_{2}$ targets, Phys. Rev. C 56 (1997) 388.

[9] C. La Tessa, S. Guetersloh, L. Heilbronn, J. Miller, L. Sihver, C. Zeitlin, Fragmentation of 1 $\mathrm{GeV} /$ nucleon iron ions in thick targets relevant for space exploration, Adv. Space Res. 35 (2005) 223-229.

[10] C. Zeitlin, A. Fukumura, L. Heilbronn, Y. Iwata, J. Miller and T. Murakami, Fragmentation cross sections of $600 \mathrm{MeV} / \mathrm{amu}{ }^{20} \mathrm{Ne}$ on elemental targets, Phys. Rev. C 64 (2001) 024902.

[11] C. Zeitlin, A. Fukumura, L.H. Heilbronn, S.B. Guetersloh, Y. Iwata, J. Miller, and T. Murakami, Fragmentation Cross Sections of ${ }^{28} \mathrm{Si}$ at Beam Energies from 290 to 1200 MeV/nucleon, LBNL Report LBNL-47655 (2006). (submitted to Nuclear Physics B).

[12] C. La Tessa, L. Sihver, C. Zeitlin, J. Miller, S. Guetersloh, L. Heilbronn, D. Mancusi, Y. Iwata, and T. Murakami, Tests of weak and strong factorization in nucleus-nucleus collisions at several hundred MeV/nucleon, (submitted to Physical Review C) (2006). 
[13] C.J. Zeitlin, K.A. Frankel, W. Gong, L. Heilbronn, E.J. Lampo, R. Leres, J. Miller and W. Schimmerling, A Modular Solid State Detector for Measuring High energy Heavy Ion Fragmentation near the Beam Axis, Rad. Meas. 23 (1994) 65-81.

[14] F. H. Attix, Introduction to Radiological Physics and Radiation Dosimetry, John Wiley \& Sons, New York (1986).

[15] F. A. Cucinotta, H. Wu, M.R. Shavers and K. George, Radiation Dosimetry and Biophysical Models of Space Radiation Effects, Gravitational and Space Biology Bulletin 16 (2003) 11-18.

[16] International Commission on Radiological Protection, Report 60, 1990 Recommendations of the International Commission of Radiation Protection, Annals of the ICRP, 21, (1991).

[17] C. Zeitlin, J. Miller, L. Heilbronn, K. Frankel, W. Gong and W. Schimmerling, The Fragmentation of $510 \mathrm{MeV} /$ nucleon Iron-56 in Polyethylene. I., Fragment Fluence Spectra, Radiat. Res. 145 (1996) 655-665.

[18] C. Zeitlin, L Heilbronn, J. Miller, Detailed Characterization of the $1087 \mathrm{MeV} /$ nucleon iron56 beam used for radiobiology at the alternating gradient synchrotron, Radiat. Res. 146 (1998) $560-569$.

[19] M. Durante, K. George, G. Gialanella, G. Grossi, C. La Tessa, L. Manti, J. Miller, M. Pugliese, P. Scampoli, and F.A. Cucinotta, Cytogenetic Effects of High-Energy Iron Ions: Dependence on Shielding Thickness and Material, Radiat. Res. 164 (2005) 571-576.

[20] C. Zeitlin, L. Heilbronn, J. Miller, W. Schimmerling, L. W. Townsend, R. K. Tripathi and J.W. Wilson, The Fragmentation of $510 \mathrm{MeV} /$ nucleon Iron-56 in Polyethylene. II., Comparisons between Data and a Model, Radiat. Res. 145 (1996) 666-672.

[21] J. W. Wilson, J. L. Shinn, L. W. Townsend, R. K. Tripathi, F. F. Badavi, S. Y. Chun, NUCFRG2: a Semiempirical Nuclear Fragmentation Model, Nucl. Instrum. Methods, Phys Rev $B, 94$ (1994) 95-102.

[22] S. A. Walker, J. W. Wilson, F. A. Cucinotta, R. K. Tripathi, S. Blattnig, C. Zeitlin, L. Heilbronn, J. Miller, Validation of the HZETRN code for laboratory exposures with $1 \mathrm{~A} \mathrm{GeV}$ iron ions in several targets, Adv. Space. Res. 35 (2005) 202-207.

[23] T. Sato, L. Sihver, H. Iwase, H. Nakashima, K. Niita, Simulations of an accelerator-based shielding experiment using the particle and heavy-ion transport code system PHITS, Adv. Space. Res. 35 (2005) 208-213.

[24] C. Zeitlin, L. Heilbronn, and J. Miller, Detailed characterization of the $1087 \mathrm{MeV} /$ nucleon Iron-56 beam used for radiobiology at the Alternating Gradient Synchrotron, Radiat. Res. 149 (1998) 560-569. We refer to the inset in Fig. 3. 
[25] H. Iwase, K. Niita, and T. Nakamura, Development of General-Purpose Particle and Heavy Ion Transport Monte Carlo Code, J. Nucl. Sci. Technol. 39 (2002) 1142.

[26] G.D. Badhwar and P.M. O'Neill, An Improved model of GCR for space exploration missions, Nucl. Tracks Radiat. Meas. 20 (1992) 403-410.

[27] H. Aiginger, et al., The FLUKA code: new developments and application to $1 \mathrm{GeV} / \mathrm{n}$ iron beams, Adv. Space Res. 35 (2005) 214-222. 
Table 1.

Beam Ions and Energies Used

\begin{tabular}{|c|c|c|c|c|c|}
\hline $\begin{array}{c}\text { Energy At Extraction } \\
(\mathrm{MeV} / \mathrm{amu})\end{array}$ & & & & & \\
\hline 290 & ${ }^{12} \mathrm{C}$ & ${ }^{14} \mathrm{~N}$ & ${ }^{20} \mathrm{Ne}$ & & \\
\hline 400 & ${ }^{12} \mathrm{C}$ & ${ }^{14} \mathrm{~N}$ & ${ }^{20} \mathrm{Ne}$ & ${ }^{28} \mathrm{Si}$ & ${ }^{40} \mathrm{Ar}$ \\
\hline 600 & ${ }^{16} \mathrm{O}$ & ${ }^{20} \mathrm{Ne}$ & ${ }^{28} \mathrm{Si}$ & ${ }^{40} \operatorname{Ar}(650)$ & ${ }^{56} \mathrm{Fe}$ \\
\hline 800 & ${ }^{28} \mathrm{Si}$ & ${ }^{56} \mathrm{Fe}$ & & & \\
\hline 1000 & ${ }^{16} \mathrm{O}$ & ${ }^{28} \mathrm{Si}(1200)$ & ${ }^{48} \mathrm{Ti}$ & ${ }^{56} \mathrm{Fe}$ & \\
\hline
\end{tabular}

Table 2.

Event- and Dose-averaged LET and Percent Dose Reduction Behind $2.83 \mathrm{~g} \mathrm{~cm}^{-2}$ Polyethylene. (Grouped by projectile species.)

\begin{tabular}{|c|c|c|c|c|c|c|}
\hline $\begin{array}{l}\text { Ion / energy } \\
\text { (MeV/amu) }\end{array}$ & $\begin{array}{l}\text { Surviving } \\
\text { Primary } \\
\text { (\%) }\end{array}$ & $\begin{array}{c}\text { Incident } \\
\text { LET } \\
(\mathrm{keV} / \mu \mathrm{m})\end{array}$ & $\begin{array}{c}\text { Event } \\
\text { Averaged } \\
L E T \\
(\mathrm{keV} / \mu \mathrm{m})\end{array}$ & $\begin{array}{c}\text { LET of } \\
\text { Surviving } \\
\text { Primaries } \\
(\mathrm{keV} / \mu \mathrm{m})\end{array}$ & $\begin{array}{c}\text { Dose } \\
\text { Averaged } \\
L E T \\
(\mathrm{keV} / \mu \mathrm{m})\end{array}$ & $\begin{array}{l}\text { Percent } \\
\text { Dose } \\
\text { Reduction } \\
\left(\mathrm{g} \mathrm{cm}^{-2}\right)^{-1}\end{array}$ \\
\hline${ }^{56} \mathrm{Fe} \quad(600)$ & 66.7 & 174.1 & 160.0 & 184.8 & 181.7 & 2.9 \\
\hline${ }^{56} \mathrm{Fe} \quad(800)$ & 69.7 & 157.9 & 139.8 & 163.1 & 154.9 & 3.9 \\
\hline${ }^{56} \mathrm{Fe}(1000)$ & 73.6 & 150.6 & 131.3 & 152.6 & 147.9 & 4.5 \\
\hline${ }^{48} \mathrm{Ti}$ (1000) & 72.1 & 107.5 & 92.4 & 108.9 & 104.2 & 5.0 \\
\hline${ }^{40} \mathrm{Ar}(400)$ & 76.6 & 99.0 & 102.2 & 109.6 & 110.8 & -1.1 \\
\hline${ }^{40} \mathrm{Ar} \quad(650)$ & 74.8 & 81.2 & 73.0 & 84.6 & 80.5 & 3.6 \\
\hline${ }^{28} \mathrm{Si} \quad(400)$ & 78.4 & 59.9 & 58.6 & 65.1 & 64.6 & 0.8 \\
\hline${ }^{28} \mathrm{Si} \quad(600)$ & 77.2 & 50.5 & 45.4 & 51.9 & 50.3 & 3.6 \\
\hline${ }^{28} \mathrm{Si} \quad(800)$ & 78.5 & 45.8 & 40.6 & 46.6 & 44.9 & 4.0 \\
\hline${ }^{28} \mathrm{Si}(1200)$ & 77.4 & 42.2 & 36.2 & 42.2 & 41.0 & 5.1 \\
\hline${ }^{20} \mathrm{Ne}(290)$ & 82.2 & 36.1 & 36.6 & 40.8 & 39.6 & -0.5 \\
\hline${ }^{20} \mathrm{Ne}(400)$ & 82.6 & 30.6 & 29.1 & 32.2 & 31.6 & 1.7 \\
\hline${ }^{20} \mathrm{Ne}(600)$ & 81.8 & 25.8 & 23.4 & 26.2 & 25.6 & 3.3 \\
\hline $\begin{array}{ll}{ }^{16} 0 & (600)\end{array}$ & 84.0 & 16.5 & 15.1 & 16.7 & 16.4 & 3.0 \\
\hline${ }^{16} 0(1000)$ & 84.3 & 14.3 & 12.7 & 14.3 & 13.9 & 3.9 \\
\hline${ }^{14} \mathrm{~N}(290)$ & 89.0 & 17.5 & 17.4 & 19.1 & 18.7 & 0.3 \\
\hline${ }^{14} \mathrm{~N}(400)$ & 85.9 & 15.0 & 14.3 & 15.5 & 15.3 & 1.7 \\
\hline${ }^{12} \mathrm{C}(290)$ & 89.3 & 13.1 & 13.1 & 14.2 & 13.9 & 0.2 \\
\hline${ }^{12} \mathrm{C}(400)$ & 87.2 & 11.0 & 10.6 & 11.3 & 11.1 & 1.4 \\
\hline
\end{tabular}


Table 3.

Radiological Protection Properties of ions Behind $2.83 \mathrm{~g} \mathrm{~cm}^{-2} \mathrm{CH}_{2}$. (Grouped by projectile species.)

\begin{tabular}{|c|c|c|c|c|c|c|c|c|}
\hline $\begin{array}{c}\text { lon / } \\
\text { energy } \\
\text { (MeV/amu) }\end{array}$ & $\begin{array}{c}\text { Dose w/o } \\
\text { Shield } \\
\text { (nGy) }\end{array}$ & $\begin{array}{c}\text { Dose with } \\
\text { Shield } \\
\text { (nGy) }\end{array}$ & $Q_{\text {in }}$ & $Q_{a v}$ & $\begin{array}{l}\text { Q Surviving } \\
\text { Primaries }\end{array}$ & $\begin{array}{l}\Delta Q \\
(\%)\end{array}$ & $\begin{array}{l}\text { H w/o } \\
\text { Shield } \\
\text { (nSv) }\end{array}$ & $\begin{array}{c}\text { H With } \\
\text { Shield } \\
\text { (nSv) }\end{array}$ \\
\hline${ }^{56} \mathrm{Fe}(600)$ & 278.9 & 256.3 & 22.8 & 22.2 & 21.8 & 2.8 & 6331 & 5639 \\
\hline${ }^{56} \mathrm{Fe}(800)$ & 252.0 & 224.0 & 23.9 & 23.5 & 23.5 & 1.5 & 6023 & 5264 \\
\hline${ }^{56} \mathrm{Fe}(1000)$ & 241.3 & 210.3 & 24.4 & 24.2 & 24.3 & 1.1 & 5888 & 5089 \\
\hline${ }^{48} \mathrm{Ti}(1000)$ & 172.2 & 148.1 & 28.9 & 27.6 & 28.7 & 5.8 & 4977 & 4088 \\
\hline${ }^{40} \mathrm{Ar} \quad(400)$ & 158.6 & 163.7 & 29.5 & 27.3 & 28.7 & 6.0 & 4679 & 4469 \\
\hline${ }^{40} \mathrm{Ar} \quad(650)$ & 130.1 & 117.5 & 23.8 & 23.6 & 24.9 & 0.6 & 3096 & 2773 \\
\hline${ }^{28} \mathrm{Si} \quad(400)$ & 96.0 & 93.9 & 17.0 & 18.4 & 18.6 & -7.6 & 1632 & 1728 \\
\hline${ }^{28} \mathrm{Si} \quad(600)$ & 80.8 & 72.8 & 14.0 & 13.9 & 14.4 & 0.3 & 1131 & 1012 \\
\hline${ }^{28} \mathrm{Si} \quad(800)$ & 73.4 & 65.0 & 12.5 & 12.2 & 12.7 & 2.3 & 918 & 793 \\
\hline${ }^{28} \mathrm{Si}(1200)$ & 67.6 & 58.0 & 11.3 & 11.0 & 11.3 & 3.4 & 764 & 638 \\
\hline${ }^{20} \mathrm{Ne}(290)$ & 57.4 & 58.6 & 9.4 & 10.5 & 10.9 & -11.7 & 540 & 615 \\
\hline${ }^{20} \mathrm{Ne}(400)$ & 49.0 & 46.6 & 7.6 & 7.9 & 8.1 & -4.4 & 372 & 368 \\
\hline${ }^{20} \mathrm{Ne}(600)$ & 41.3 & 37.4 & 6.1 & 6.0 & 6.2 & 0.6 & 252 & 224 \\
\hline${ }^{16} \mathrm{O} \quad(600)$ & 26.4 & 24.2 & 3.1 & 3.1 & 3.1 & 0.0 & 81.8 & 75.0 \\
\hline${ }^{16} \mathrm{O}(1000)$ & 22.8 & 20.3 & 2.4 & 2.3 & 2.4 & 2.0 & 54.7 & 46.7 \\
\hline${ }^{14} \mathrm{~N} \quad(290)$ & 28.1 & 27.8 & 3.4 & 3.8 & 3.9 & -10.5 & 95.5 & 105.6 \\
\hline${ }^{14} \mathrm{~N} \quad(400)$ & 24.0 & 22.8 & 2.6 & 2.8 & 2.8 & -5.5 & 62.4 & 63.8 \\
\hline${ }^{12} \mathrm{C} \quad(290)$ & 20.1 & 20.1 & 2.0 & 2.3 & 2.3 & -14.9 & 42.0 & 48.3 \\
\hline${ }^{12} \mathrm{C} \quad(400)$ & 17.6 & 16.9 & 1.3 & 1.4 & 1.4 & -7.6 & 22.9 & 23.7 \\
\hline
\end{tabular}




\section{FIGURE CAPTIONS}

1. Schematic diagram of silicon detector stack used for shielding analysis. A trigger detector TR and $\mathrm{d} 3 \mathrm{mmU}$ were mounted upstream of the target to identify the species and define the location of particles incident on the target. Downstream of the shield were several silicon detectors arranged in pairs, each subtending slightly different angles of acceptance. Also shown (but not used in the analysis presented here) are the plastic Timeof-Flight scintillators and a sodium iodide crystal used to stop the heavier ions.

2. Comparison of fragmentation properties of aluminum and polyethylene $\left(\mathrm{CH}_{2}\right)$. Roughly equal areal densities of $\mathrm{CH}_{2}\left(4.5 \mathrm{~g} \mathrm{~cm}^{-2}\right)$ and aluminum $\left(5.0 \mathrm{~g} \mathrm{~cm}^{-2}\right)$ were used to shield an incident beam of $1 \mathrm{GeV} / \mathrm{amu}{ }^{56} \mathrm{Fe}$. For a given areal density, $\mathrm{CH}_{2}$ causes more fragmentation of the incident iron ions than does aluminum.

3. Percent dose reduction as a function of shielding thickness, for $1 \mathrm{GeV} / \mathrm{amu}{ }^{16} \mathrm{O}$ incident on polyethylene. This quantity remains fairly linear (dahed line) to a thickness of about 4 or $5 \mathrm{~cm}$ of $\mathrm{CH}_{2}$. The error bars reflect the estimated $\pm 5 \%$ uncertainties discussed in the text.

4. Percent dose reduction, normalized to the areal density, for the same shield for different ion/energy combinations. All data were taken behind a $2.83 \mathrm{~g} / \mathrm{cm}^{2}$ polyethylene target. Solid lines connect projectile ions having equal (or nearly equal) energies when incident on the target. Experimental uncertainties are indicated by the sizes of the plot symbols.

5. Measured values of $\delta \mathrm{D}_{\mathrm{n}}$ and $\Delta Q$ (see the text for definitions of these quantities), plotted against values predicted by Monte Carlo calculations made with the BBFRAG code. The Monte Carlo reproduces the general trends seen in the data, but the lack of non-leading fragments in the calculation leads to small discrepancies.

6. Monte Carlo calculations using BBFRAG of Bragg curves in $\mathrm{CH}_{2}$ for $1 \mathrm{GeV} / \mathrm{amu}{ }^{56} \mathrm{Fe}$ (Fig. $7 \mathrm{a}$ at top) and $290 \mathrm{MeV} / \mathrm{amu}{ }^{28} \mathrm{Si}$ (Fig 7b, bottom). In both examples, three quantities are plotted as functions of depth: primary LET (black curves), dose-averaged LET (red curves), and event-averaged LET (blue curves). For relatively high-energy particles as in Fig. 7a, fragmentation is the dominant effect in the depth-dose relation; at lower energies, as in Fig. 7b, the effect of ionization energy loss dominates. At intermediate energies, the two effects often balance each other, or nearly so, resulting in flat Bragg curves over the first several $\mathrm{g} \mathrm{cm}^{-2}$ of depth.

7. Calculated dose due to neutrons produced in $\mathrm{CH}_{2}$ shielding from $1 \mathrm{GeV} / \mathrm{amu}{ }^{56} \mathrm{Fe}$. The neutron dose increases as a function of depth until the range of the projectile ion is reached. The calculation was performed with the PHITS Monte Carlo code.

8. BBFRAG simulation of Galactic Cosmic Rays incident on varying depths of $\mathrm{CH}_{2}$, showing average LET and average quality factor. Only charged particles are simulated, and the multiplicity is underestimated in the model, so the average LET results are not 
expected to be accurate at the larger depths. Nonetheless, it can be seen that fragmentation and stopping of incident heavy ions cause both LET and average quality factor to steadily decrease with depth, asymptotically approaching the values of 0.2 $\mathrm{keV} / \mu \mathrm{m}$ and 1 , respectively, corresponding to minimum-ionizing particles. 


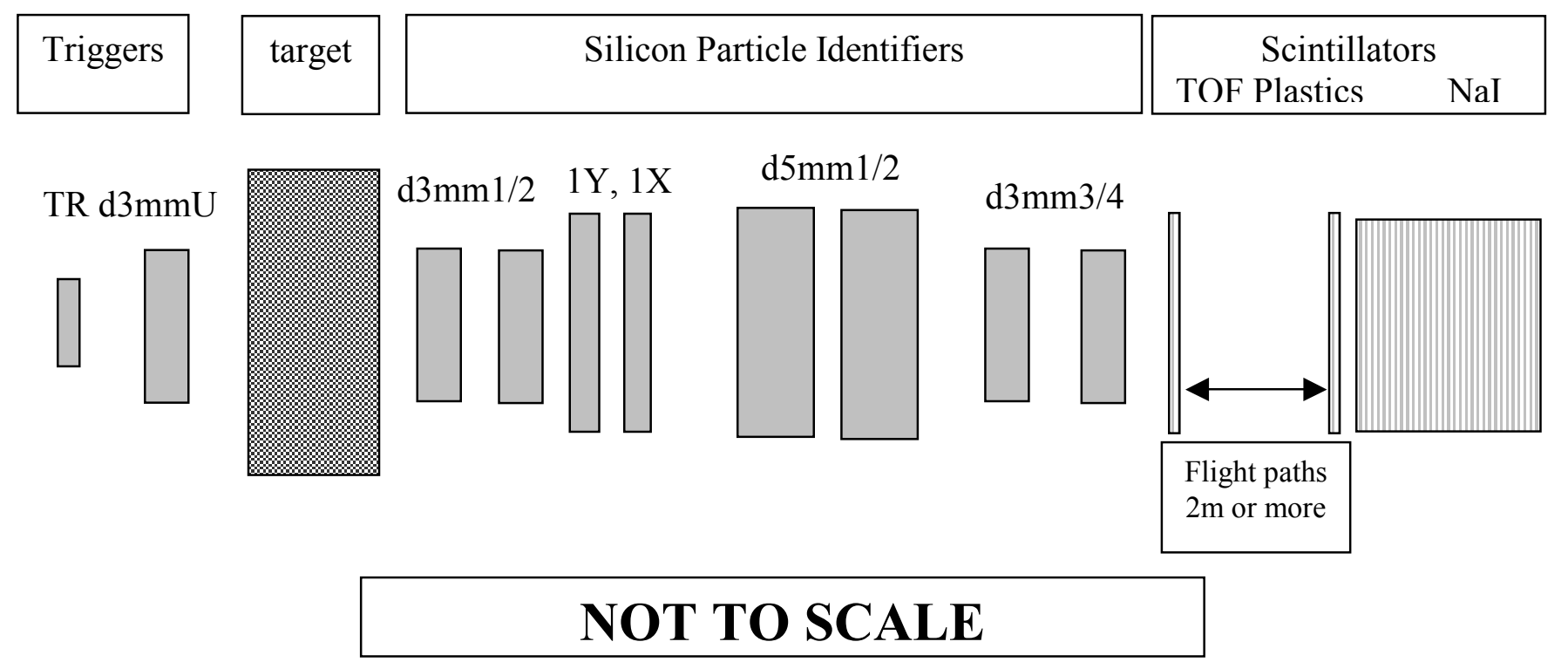

Figure 1 


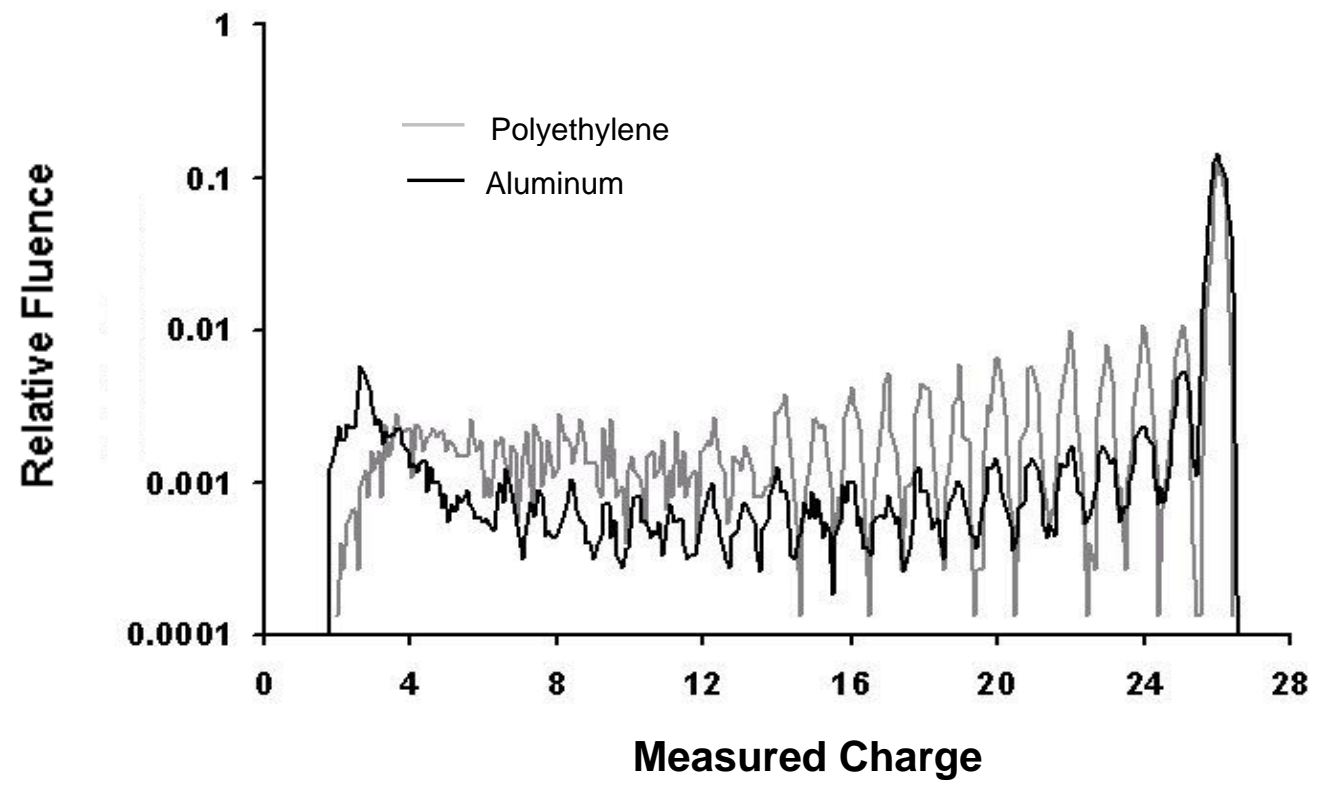

Figure 2 


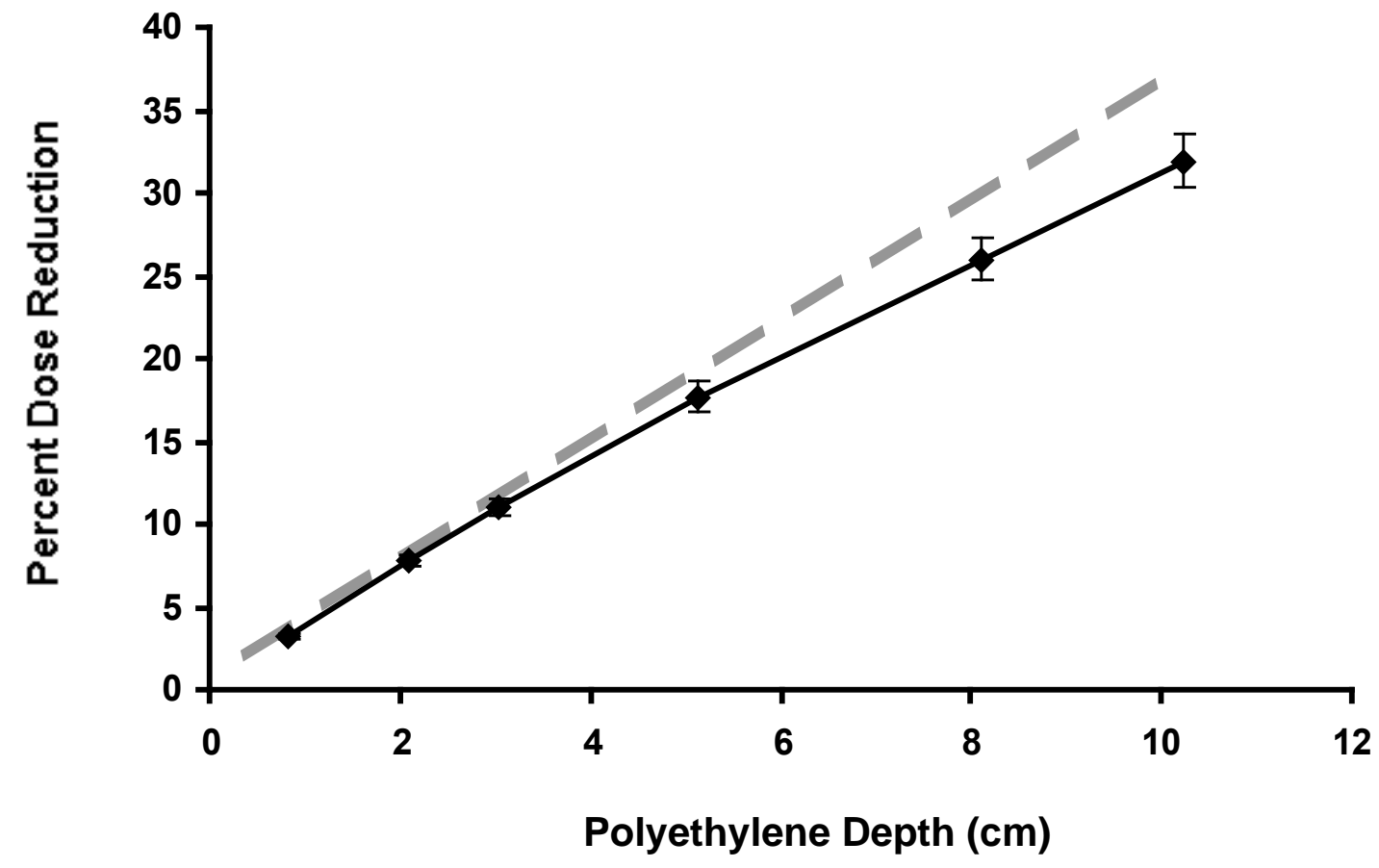

Figure 3 


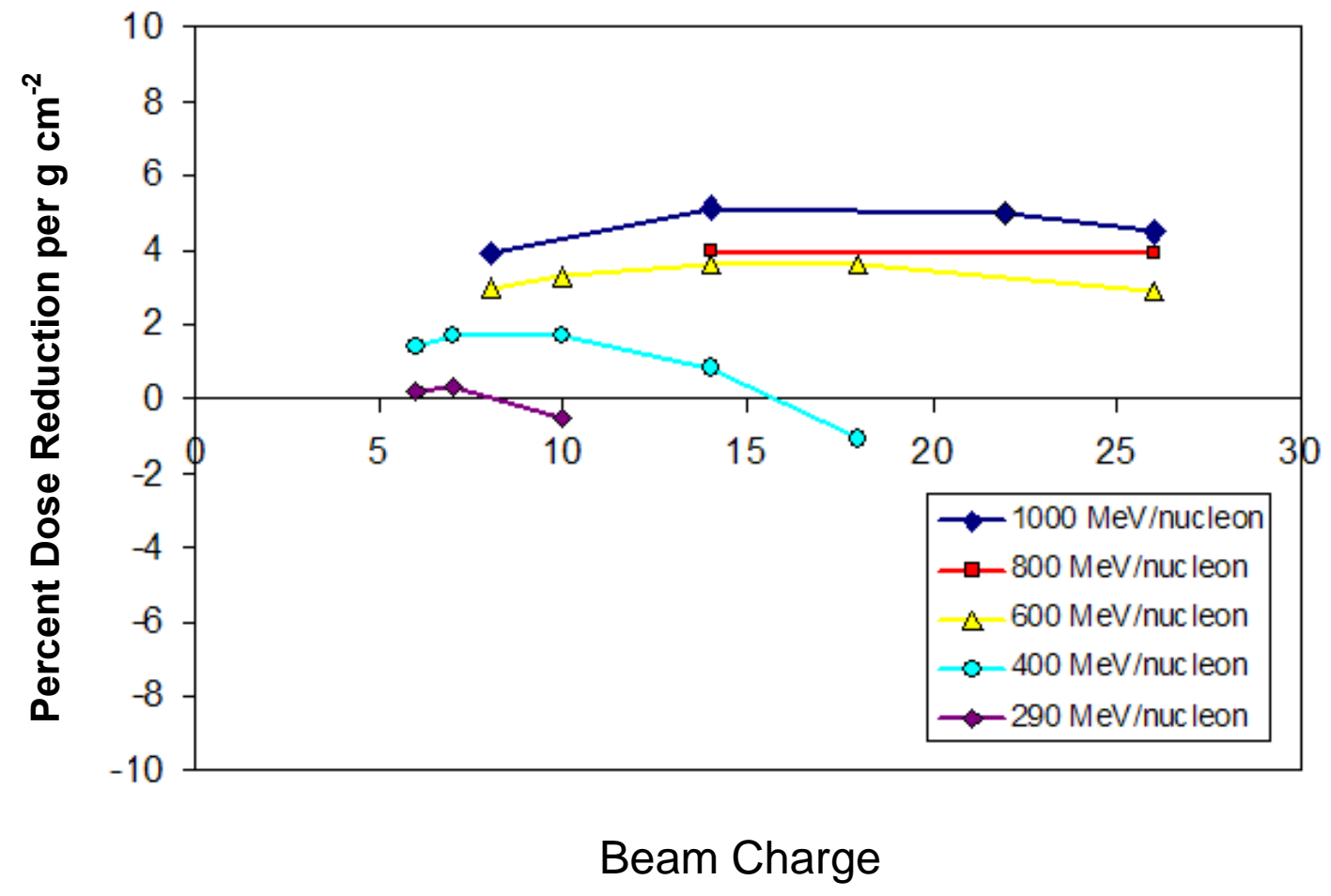

Figure 4 


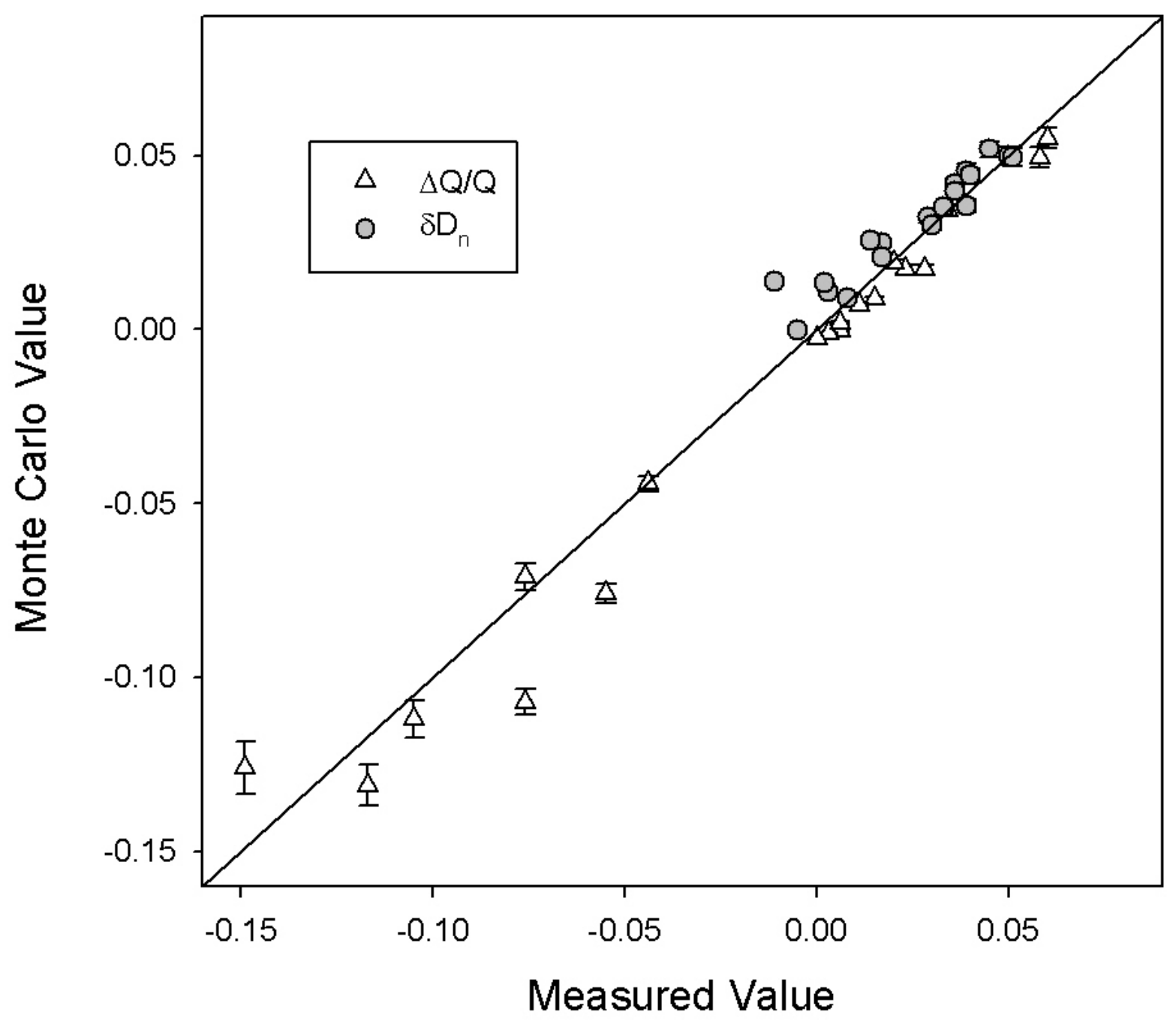

Figure 5 
Figure 6

Monte Carlo for $1 \mathrm{GeV} / \mathrm{amu}{ }^{56} \mathrm{Fe}$

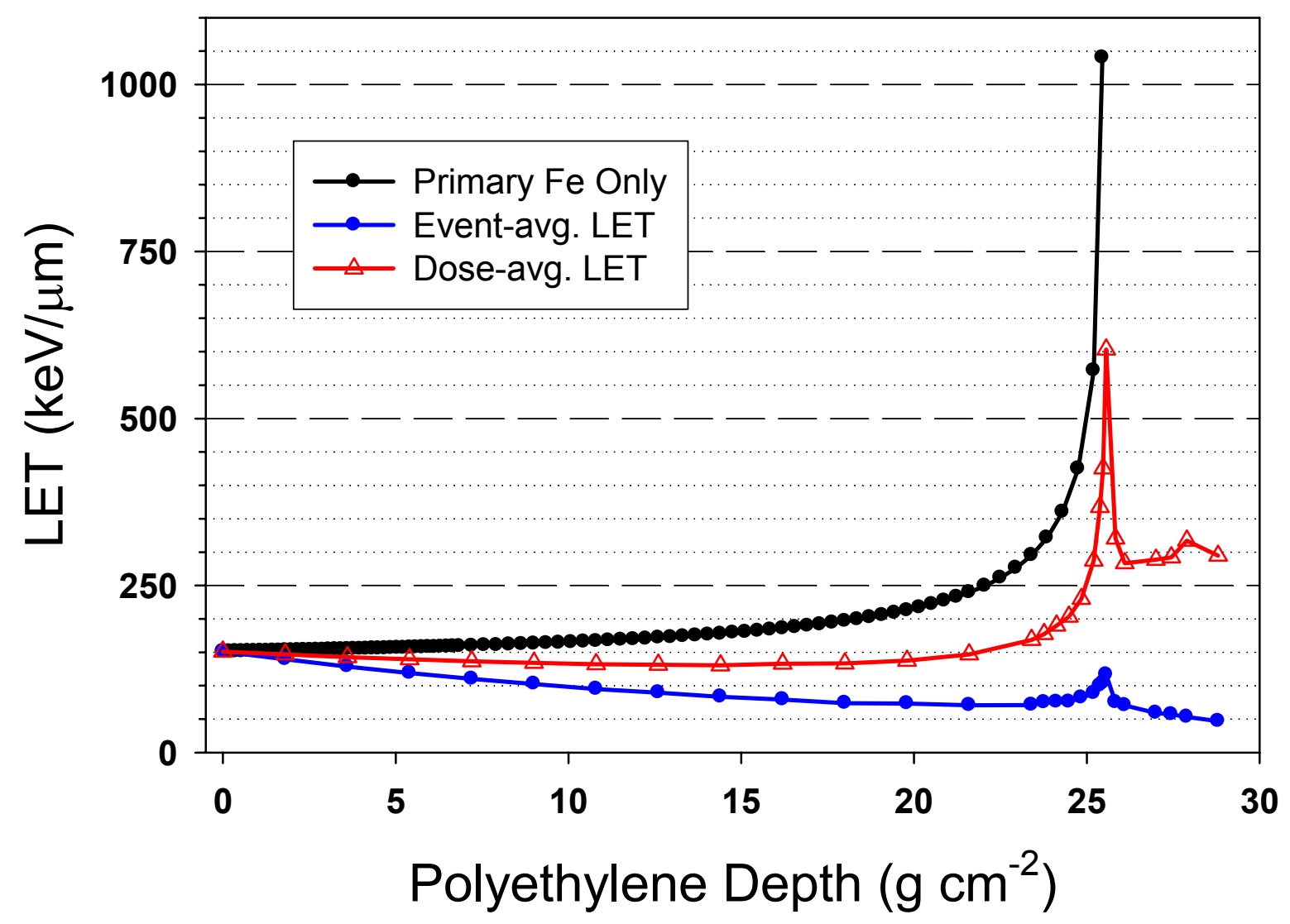

$6 b$

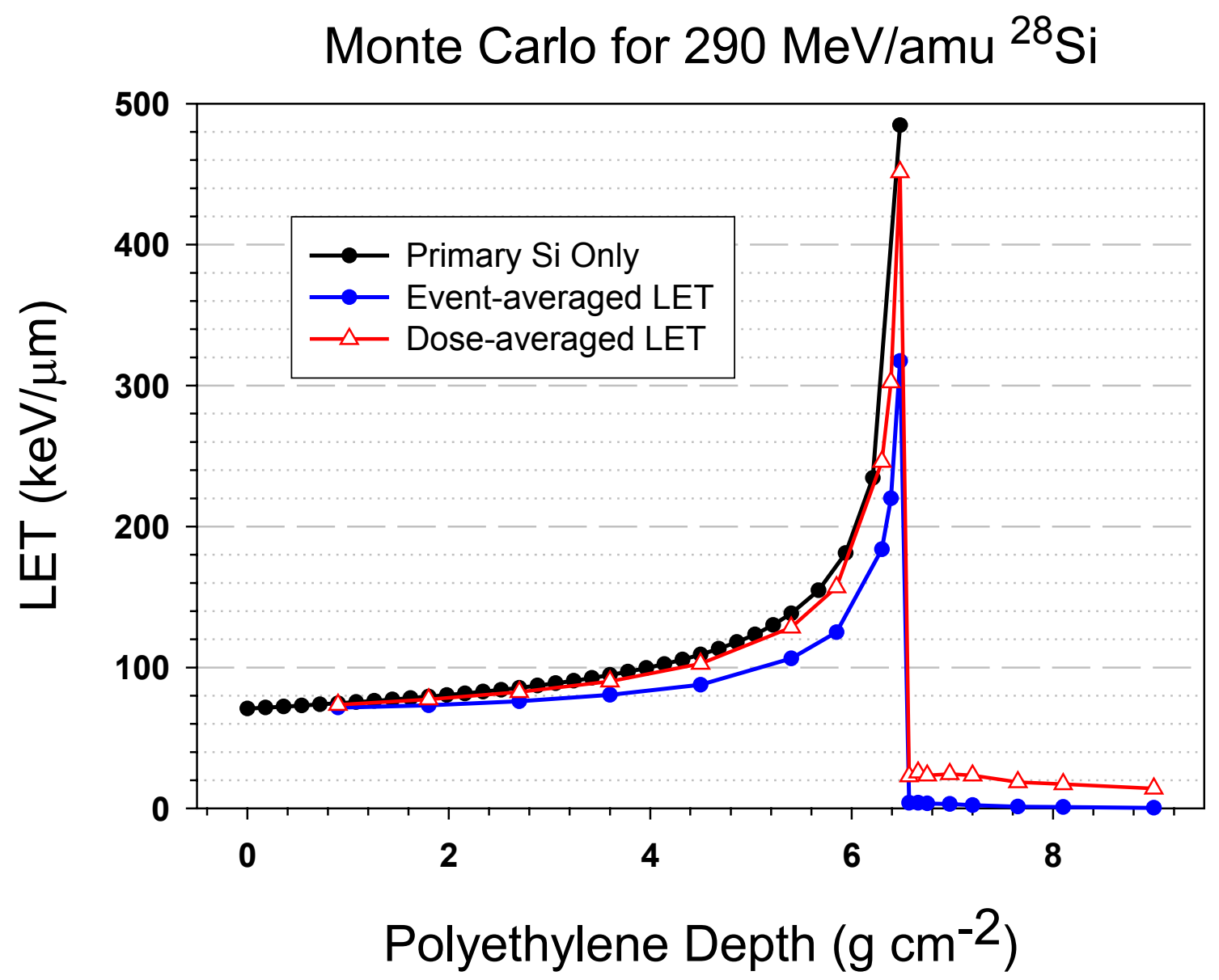




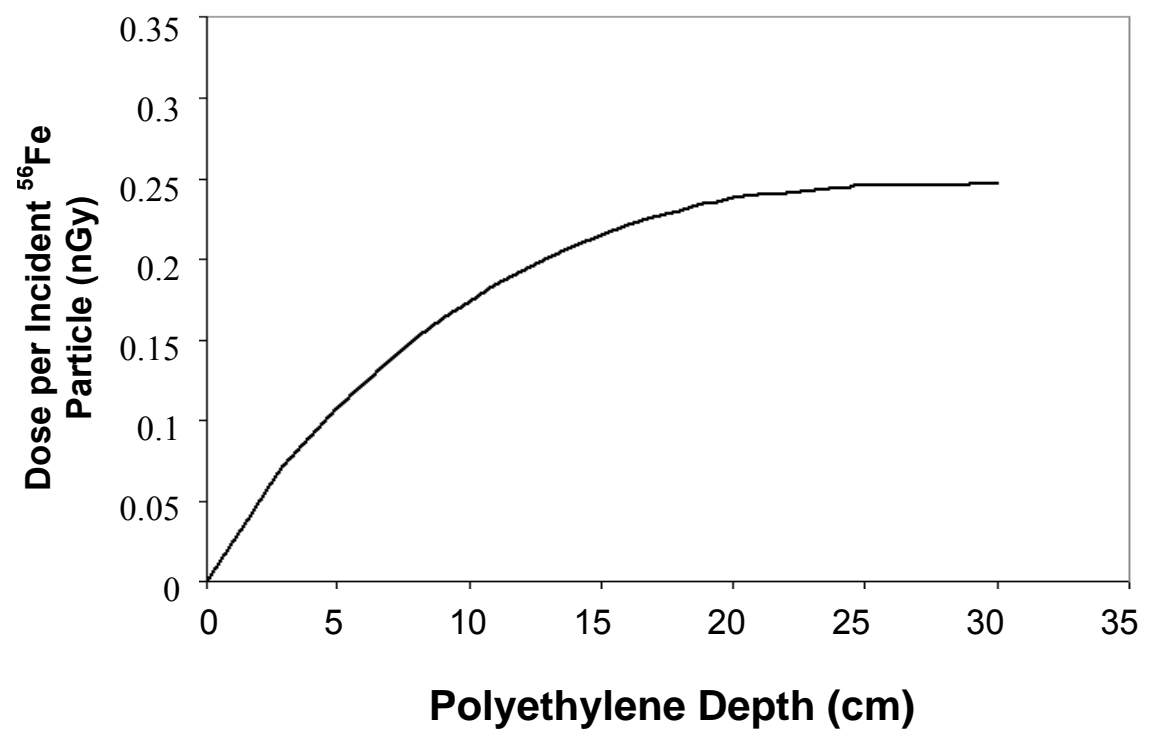

Figure 7 
Monte Carlo GCR Simulation Charged Particles Only

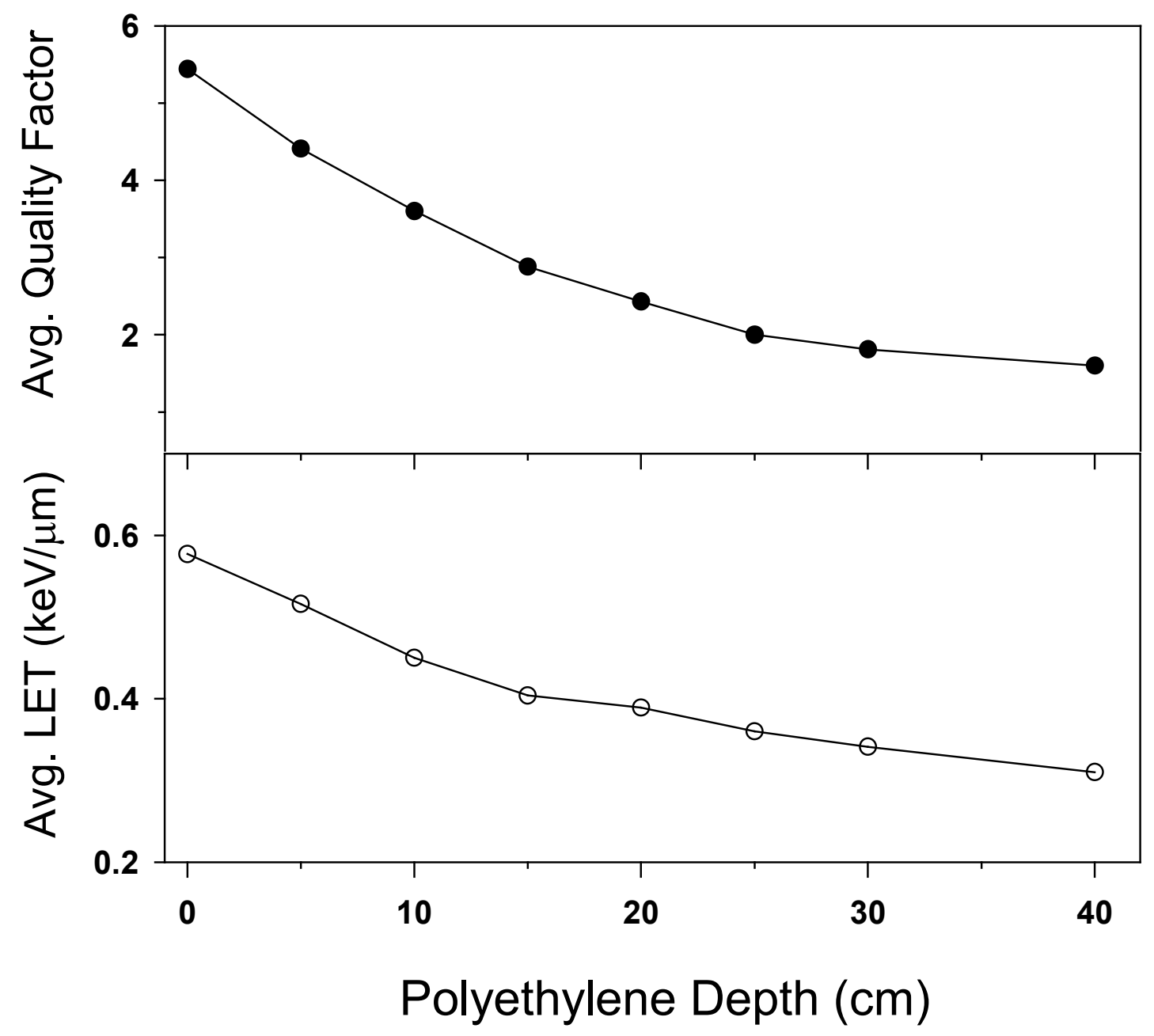

Figure 8 